\title{
Movilidad residencial y (re)composición social del espacio urbano en el municipio de Madrid*
}

\author{
Andrea Andújar Llosa \\ Universidad Pablo de Olavide \\ aandllo@upo.es
}

Recepción: 24-01-2017

Aceptación: 14-07-2017

\section{Resumen}

La importancia de la movilidad ha sido, explícita o implícitamente declarada, esencial en los estudios relacionados con la composición social del espacio urbano y las transformaciones asociadas a la misma. Proponiendo un marco interpretativo desde la sociología urbana, este artículo analiza el efecto de la movilidad residencial en la transformación de la composición social del espacio urbano a escala inframunicipal en los periodos 2000-2007 y 2008-2013 en el municipio madrileño. Los resultados muestran que los efectos de la movilidad no son simples ni directos, por lo que resulta necesario atender a los cambios producidos en los agentes sociales protagonistas, a las modificaciones de las prácticas residenciales y a su relación con el espacio. El análisis evidencia un cambio en los efectos de la movilidad entre ambos periodos y la coexistencia de distintos procesos relacionados con la movilidad, lo que muestra su potencial como elemento analítico e interpretativo de los procesos de transformación urbana de carácter más general.

Palabras clave: localización residencial; transformación urbana; barrios; posición social; prácticas socioespaciales

* Este texto se ha desarrollado en el marco del contrato predoctoral FPI BES-2012-059276 financiado por el Programa Nacional de Formación de Recursos Humanos, Subprograma de Formación de Personal Investigador del Ministerio de Economía y Competitividad, asociado al proyecto Dinámicas y transformaciones territoriales, funcionales y sociales de las áreas metropolitanas españolas en un horizonte de sostenibilidad (METROSOST) (CSO201455780-C3-1-P). 
Abstract. Residential Mobility and the Social Recomposition of Urban Space in the Municipality of Madrid

Explicitly or implicitly, the relevance of mobility has been declared essential in studies on the social composition of urban space and the transformations associated with it. This article proposes an interpretive, urban sociology framework to analyse the effect of residential mobility in the transformation of the social composition of urban space at neighbourhood scale in the municipality of Madrid for the time periods 2000-2007 and 2008-2013. The results show that the effects of mobility are neither simple nor direct, thus making it necessary to address the changes produced in the main social agents, their residential practices and their relationship with urban space. The analysis shows a change in the effects of mobility between the two periods and the coexistence of different processes related to mobility, thus revealing their potential as an analytical and interpretive element of urban transformation processes of a more general nature.

Keywords: residential location; urban transformation; neighbourhoods; social position; socio-spatial practices

\section{Sumario}

1. Introducción

2. La movilidad residencial en los procesos de transformación urbana

3. Metodología

4. La interpretación territorial a partir de las prácticas socioespaciales
5. Procesos manifestados a través de la movilidad residencial

6. Conclusiones

Referencias bibliográficas

Anexo

\section{Introducción}

La movilidad residencial supone un componente esencial en los procesos de localización, segregación o composición social del espacio urbano. Desde los modelos de invasión y sucesión desarrollados por los primeros estudios de la Escuela de Chicago hasta algunos de los principales debates actuales, se centran en el papel de la movilidad residencial en la dinámica de la composición social de la ciudad.

Este artículo tiene como objetivo analizar los efectos de la movilidad residencial en la composición social del espacio urbano, es decir, en las características sociales de quienes residen en un determinado espacio. ¿Contribuye la movilidad residencial a reproducir la composición social del espacio urbano o contribuye a su transformación?

El estudio de los efectos de la movilidad residencial en los procesos de transformación urbana ha estado dominado por las aproximaciones centradas en las decisiones de individuos y hogares, lo que ha eclipsado el desarrollo de marcos interpretativos que permitan analizar la movilidad residencial como práctica social configurada por condiciones estructurales y objetivas. Aquí se propone realizar una interpretación de la movilidad residencial como una 
práctica socioespacial doblemente estructurada (social y espacialmente), por lo que estará condicionada tanto por la posición social ocupada como por la estructura urbana desigual y jerárquica. Desde esta interpretación, se desprende la hipótesis de que la movilidad residencial, en sí misma, tenderá a reproducir la división social del espacio urbano, pero que, sin embargo, esta es clave en procesos de transformación urbana de carácter más general.

A pesar de que el estudio de la movilidad residencial supone una herramienta útil para analizar la forma en que las prácticas y los procesos sociales interaccionan con el territorio (Módenes, 1998), los estudios a nivel inframunicipal sobre los efectos de la misma en la dinámica urbana son todavía escasos en España ${ }^{1}$, donde la precariedad de las fuentes estadísticas dificulta su desarrollo.

Este artículo presenta una aproximación novedosa al estudio del papel de la movilidad residencial en la composición social del espacio urbano en los barrios del municipio madrileño. Se trata de una exploración a partir de la información padronal que, aunque limitada en las variables que recoge, permite contar con un nivel de análisis desagregado temporal y espacialmente a partir de las altas y las bajas padronales. Para ello, se realiza, como propone Di Virgilio (2011) retomando a Lefebvre, una «lectura del espacio por medio del examen de las "prácticas espaciales"” a partir de una tipología de los barrios relacionando los tipos de movimientos (entradas, salidas y movimientos internos) con su intensidad. Posteriormente, se analiza la relación estadística entre la intensidad de la movilidad y diferentes indicadores de transformación sociodemográfica. Si bien la información padronal limita las conclusiones en relación con la composición social del espacio urbano, el análisis evidencia resultados relevantes y abre nuevas preguntas de investigación.

\section{La movilidad residencial en los procesos de transformación urbana}

La interpretación del papel que desempeña la movilidad residencial en la división social del espacio, así como en sus transformaciones, se enmarca en un debate todavía presente en los estudios urbanos. Así, se manifiesta una distinción entre aquellas aproximaciones que enfatizan en la capacidad de agencia de los individuos, que interpretan la movilidad residencial como la suma de comportamientos individuales que determinan la configuración de la demanda residencial, y aquellas que focalizan en los elementos estructurales en los procesos de transformación urbana.

Desde los trabajos pioneros de Rossi (1955) y Brown y Moore (1970), el análisis de las decisiones y de las elecciones de los hogares ha tenido una importante influencia en la interpretación de la relación entre los desplazamientos residenciales y los procesos de configuración de la composición social del espacio urbano. Desde esta perspectiva, la suma de comportamientos individuales

1. Esta ha sido más analizada en el caso español en los procesos relacionados con la configuración metropolitana a nivel municipal (Pujadas Rubies, 2005 y 2009; Susino, 2003 y 2010; Feria, 2008 y 2010), pero escasamente estudiada empíricamente a nivel inframunicipal. 
- con gran importancia dada a los procesos de ajuste entre el hogar y la vivienda (Rossi, 1955; Clark y Dieleman, 1996; Clark y Onaka, 1983; Clark, 2012) producidos a lo largo del curso de vida (Mulder, 1993; Mulder y Hooimeijer, 1999; Kendig, 1990) - es el mecanismo que reconfigura el mosaico urbano (Clark, 2012; Everaers y Maas, 1985), y son los promotores y los constructores los que se apoyan en dichas elecciones (Lévy y Brun, 2002). El aumento relativo de las clases medias y sus preferencias residenciales en determinadas fases del ciclo del hogar se encontrarían, así, en la base de procesos como la suburbanización (López et al., 2013; Susino, 2003) o la gentrificación ${ }^{2}$ (Gale, 1979; Lévy y Brun, 2002; Ley, 1996; López-Gay, 2016; Karsten, 2007). Así mismo, la movilidad residencial es el mecanismo por el que los individuos «escapan» de determinados barrios cuando tienen ocasión, mientras que aquellos más pobres quedan «atrapados» en ellos mediante un proceso de "filtrado» (Clark et al., 2014; Hedman, 2011; van Ham y Clark, 2009), lo que conforma espacios de «relegación» o «exilio» (Donzelot, 2007; Dubet y Lapeyronnie, 1992, en Vignal, 2014). En definitiva, estas aproximaciones enfatizan en la consideración de que la movilidad residencial puede transformar las dinámicas territoriales en base a las decisiones de los individuos y de los grupos sociales (Pinçon-Charlot, 1989; Grafmeyer, 1991; Authier, 2003, citados en Authier, 2014).

En contraposición, otras aproximaciones teóricas (especialmente las neoweberianas y neomarxistas) focalizan en los modos de producción de la ciudad y en los elementos estructurales como determinantes a la hora de interpretar la movilidad y los procesos de composición social del espacio urbano. Frente a la excesiva importancia del curso de vida y del proceso de mejora asociados al housing career, resulta interesante el concepto housing histories (Forrest, 1987) bajo la consideración de que las experiencias residenciales son compartidas en base a categorías sociales como la clase, la etnia, el género y la localización. En relación con los procesos de reconfiguración urbana, los modos de producción de la ciudad adquieren mayor protagonismo como elementos fundamentales en la elaboración de las opciones residenciales, para lo que se considera esencial conocer el funcionamiento de los mercados y de los sistemas residenciales (Murie, 1997; Leal, 2002), y la consideración de todos los actores con intereses económicos en el mismo (Form, 1954; Van Kempen, 2002).

La perspectiva neomarxista enfatiza en la lógica de apropiación del sistema capitalista en la generación de la desigualdad urbana, fuertemente determinada por el auge del neoliberalismo como sistema de organización y producción social (Theodore et al., 2009). Los procesos de inversión (o de abandono) se interpretan como predecesores o causantes de los movimientos de personas

2. Españolización del término gentrification. No se pretende aquí entrar en un debate en torno al concepto, que cuenta ya con un gran desarrollo teórico. Una definición sintética de esta palabra es la elaborada por Clark y rescatada por Sorando: «La gentrificación es un proceso que implica un cambio en la población de los usuarios del territorio tal que los nuevos usuarios son de un estatus socioeconómico superior al de los usuarios previos, junto con un cambio asociado en el medio construido a través de una reinversión en capital fijo» (Clark, 2005: 258, en Sorando, 2015: 214). 
(Smith, 1979), y la «movilidad residencial» es escasamente planteada como objeto de estudio. Frente a la «elección» y a las «decisiones» en la explicación de la localización residencial, emergen conceptos como el desplazamiento y la desposesión que los procesos urbanos generan en determinados grupos sociales, oponiéndose a las aproximaciones imperantes de las teorías neoclásicas basadas en las decisiones individuales constreñidas por recursos y obviando las estructuras políticas y económicas (Slater, 2013). La movilidad residencial, como uno de los comportamientos esenciales en relación con la vivienda y el espacio urbano, es interpretada más como una consecuencia que como una causa de la configuración y de la transformación de la ciudad.

Si bien las investigaciones empíricas desarrolladas desde estas perspectivas aportan claves de conocimiento de gran riqueza, los marcos interpretativos en los que se sustentan a menudo se presentan algo parciales a la hora de dar cuenta de la relación entre los elementos estructurales y del desarrollo de prácticas sociales en el espacio urbano. Con el objetivo de emplear un marco interpretativo de la movilidad residencial como práctica socioespacial, se propone aquí la aplicación de algunas de las aportaciones teóricas del estructuralismo constructivista de Pierre Bourdieu (1988). A través del reconocimiento del papel configurador de las estructuras — «que no pueden ser reducidas a las interacciones y prácticas a través de las cuales se expresan» (Bourdieu y Wacquant, 2005: 170)_, permite interpretar los comportamientos, los valores y las actitudes de los agentes sociales, no de una manera mecanicista, sino en términos de probabilidad en función de la posición que ocupan en el espacio social.

La movilidad residencial, como práctica socioespacial, se desarrolla, así, en relación con una doble estructuración. En primer lugar, se encuentra condicionada por la posición social ocupada por los agentes sociales y la estructura del capital poseído ${ }^{3}$, que actúa como factor estructurante por medio del hábitus (Bourdieu, 1988, 1997; Bourdieu y De Saint Martin, 1990). Las preferencias y las elecciones residenciales antes mencionadas son más bien disposiciones y prácticas de los agentes que no se interpretan en términos de libre elección, ni como consecuencia directa de la posición social, sino en términos de probabilidad que se incrementa cuanto mayor sea la proximidad entre las posiciones ocupadas en el espacio social ${ }^{4}$, y que se desarrollan en el marco de opciones posibles 5 (Bourdieu y De Saint Martin, 1990). De modo similar al propuesto por Forrest (1987), las prácticas residenciales estarán configuradas por la

3. El capital global del que disponen los agentes sociales está configurado por la "cuantía» de cada una de las subespecies de capital: económico, cultural, social y simbólico.

4. Se emplea el concepto de espacio social en el sentido propuesto por Bourdieu. Cada vez que se haga referencia al mismo, será en relación con la estructura social y no con la dimensión geográfica.

5. Los sistemas de disposiciones de los agentes también están configurados por el marco que establecen las opciones mínimamente realistas o realizables, en el que desempeñan un papel esencial el Estado, a través de la política de vivienda, y los agentes con capacidad de influencia en la configuración de las representaciones que los agentes sociales producen individual y colectivamente (Bourdieu y Christin, 1990; Bourdieu et al., 1990). 
posición social y las trayectorias de clase. Desde esta perspectiva, la posición social, en tanto en cuanto determina las disposiciones colectivas, supone un principio estructurador de la movilidad residencial, y no un componente más de la misma (Di Virgilio, 2014).

En segundo lugar, las prácticas residenciales se desarrollan en un espacio también jerarquizado, en una "estructura espacial de la ciudad» (Cortés, 1995: 139), resultado de una doble producción social: mediante la localización de bienes y servicios y mediante su apropiación por parte de agentes sociales que ocupan una determinada posición social (Bourdieu, 1999). Esta jerarquización cuenta con importantes inercias históricas e implica que las prácticas socioespaciales (y la movilidad residencial en concreto) se desarrollan distintivamente en función de la posición social, y se insertan desigualmente en el territorio urbano.

Entra en juego, de este modo, la agencia de los sujetos, pero una agencia determinada y configurada en un sistema de medios materiales y simbólicos diferenciados y configurados por las estructuras de relaciones objetivas. Desde esta perspectiva, los cambios residenciales pueden formar parte de las estrategias de reproducción social, pero también pueden suponer un efecto de la desposesión de capital que posibilita el acceso asegurado a la vivienda o el mantenimiento de la posición ocupada en el campo residencial, lo que dará lugar a cambios voluntarios, inducidos o forzados ${ }^{6}$.

\section{1. (Re)composición social del espacio urbano y movilidad residencial}

La definición de un espacio urbano, un hábitat (empleando el concepto más comúnmente usado en Francia y en Latinoamérica), puede ser modificada por las propiedades que lo determinan, es decir, ya sea por una modificación de las características físicas (bienes y servicios en el mismo y características de las viviendas que lo componen) o por una modificación de sus características sociales (es decir, por una modificación de la posición social de los agentes y de las instituciones que se relacionan o se apropian del mismo).

Si la apropiación del espacio urbano por parte de agentes e instituciones sociales, mediante su localización y dotación de significación social, determina parcialmente su caracterización y jerarquización, la movilidad residencial aparece como un elemento esencial para comprender la dinámica de la división social del espacio urbano ${ }^{7}$ (Duhau, 2003). Como ya se ha mencionado, la

6. Frente a la clasificación objetivista de los cambios residenciales como ascendentes, descendentes o neutros (Lévy, 1998), otras clasificaciones (Clark y Onaka, 1983; Susino, 2003) proponen la incorporación de las percepciones y de las motivaciones sobre los cambios de vivienda, distinguiendo entre aquellos que se hacen "forzados por las circunstancias, de aquellos otros que suponen una opción positiva, un proyecto» (Susino, 2003: 84).

7. Se emplea el concepto de división social del espacio urbano para hacer referencia a «las diferencias existentes en la localización intraurbana o intrametropolitana de diferentes grupos, estratos o clases sociales» (Duhau, 2003: 177). El concepto es similar al uso frecuentemente dado al de segregación residencial cuando esta se define como «el grado de proximidad espacial [...] 
movilidad residencial se desarrolla en relación con dos estructuras (social y espacial), por lo que, en sí misma, tenderá a reproducir la división social del espacio urbano existente (Sharkey, 2012). Pero si bien es cierto que esta no puede entenderse como el elemento causal último de los procesos de transformación urbana, sí supone una parte esencial de los mismos ${ }^{8}$. Teniendo en cuenta los factores que condicionan las movilidades que emprenden los agentes sociales, «la movilidad espacial puede funcionar como un poderoso analizador de las mutaciones territoriales en curso en las grandes metrópolis contemporáneas» (Dupont et al., 2002: XVII), no tanto por ser su soporte (Susino, 2003), sino por constituir un elemento esencial en la producción de la ciudad como consecuencia de la relación entre espacio físico y espacio social.

Sin embargo, la movilidad de los agentes sociales puede conllevar tanto un mantenimiento como una transformación de su composición social, en función de la combinación entre movilidad social y movilidad espacial. Un espacio altamente afectado por cambios residenciales puede relacionarse con una transformación en su composición social si quienes llegan y se van cuentan con posiciones sociales diferenciadas. Por el contrario, estos nuevos asentamientos pueden llevarse a cabo por agentes con posiciones sociales similares a aquellos que los abandonan, sin conllevar cambios en su composición social. Por otra parte, las modificaciones en ese aspecto pueden producirse por un reposicionamiento social de quienes lo habitan o, al menos en el largo plazo, por un cambio en la composición demográfica de dicha zona obligada por el mero paso del tiempo. Así, la composición social de un espacio urbano puede sufrir modificaciones mediante dos tipos de procesos: mediante un reposicionamiento en el espacio social por parte de sus habitantes (por un proceso de movilidad social intrageneracional o intergeneracional) o por un proceso de apropiación por parte de agentes sociales con una posición social diferente a la de aquellos que lo habitan originariamente (por procesos de movilidad residencial). Los efectos de la movilidad residencial dependerán, por tanto, de las particularidades de quienes se mueven, de las prácticas residenciales de distintos grupos sociales y de las características de los espacios en los que estas se insertan.

\section{Metodología}

A fin de analizar la movilidad residencial en la composición social del espacio urbano, se ha realizado un análisis que comprende dos etapas principales: en

[de] un mismo grupo social, sea que este se defina en términos étnicos, etarios, de preferencias religiosas o socioeconómicos» (Sabatini et al., 2001: 27). Sin embargo, para algunos autores, la segregación solo existe cuando es producida por medidas coercitivas (Merlin, 1998, en Duhau, 2003) o por razones étnicas (Marcuse, 2001, en Rodríguez, 2014).

8. De hecho, muchos de los procesos urbanos que se desarrollan en búsqueda de la revalorización económica a través de la dimensión urbanística, residencial, funcional o simbólica necesitan o promueven un proceso de transformación de la composición social, que, en definitiva, se materializa mediante procesos de movilidad de los agentes sociales. 
Cuadro 1. Movilidad y cambio en la composición social del espacio urbano

\begin{tabular}{|c|c|c|}
\hline & Recomposición social & No recomposición social \\
\hline \multirow{2}{*}{$\begin{array}{l}\text { Movilidad } \\
\text { residencial }\end{array}$} & Sustitución & Especialización \\
\hline & $\begin{array}{l}\text { Los agentes que llegan cuentan con } \\
\text { una posición social diferente a la de } \\
\text { aquellos originarios del lugar y/o a } \\
\text { la de aquellos que abandonan dicho } \\
\text { espacio. }\end{array}$ & $\begin{array}{l}\text { Los agentes que llegan cuentan con } \\
\text { una posición social similar a la de } \\
\text { aquellos originarios del lugar y/o a } \\
\text { la de aquellos que abandonan dicho } \\
\text { espacio. Aunque haya una alta } \\
\text { movilidad residencial, no se produce } \\
\text { un cambio en su composición social. }\end{array}$ \\
\hline \multirow{2}{*}{$\begin{array}{l}\text { Permanencia } \\
\text { residencial }\end{array}$} & Transformación por movilidad social & Mantenimiento \\
\hline & $\begin{array}{l}\text { Se produce un reposicionamiento en el } \\
\text { espacio social de quienes viven en un } \\
\text { territorio dado, pero no se cambia de } \\
\text { lugar de residencia tras él. Modificación } \\
\text { de su composición social sin movilidad } \\
\text { física. }\end{array}$ & $\begin{array}{l}\text { Estabilidad en la posición social y en el } \\
\text { lugar residencial de quienes habitan un } \\
\text { espacio urbano. En el largo plazo, esta } \\
\text { combinación no es posible. }\end{array}$ \\
\hline
\end{tabular}

Fuente: elaboración propia.

primer lugar, la elaboración de una tipología de barrios del municipio madrileño en función de la movilidad que les afecta y, en segundo lugar, un análisis de la relación de esta movilidad con diferentes indicadores de variación de composición sociodemográfica de dichos barrios.

El análisis se ha realizado en referencia a un periodo lo suficientemente amplio como para evidenciar patrones estables, además de para identificar posibles consecuencias que la crisis iniciada en 2008 pudiera haber conllevado en las prácticas residenciales de los grupos sociales. Así, el análisis se desarrolla con datos desde el año 2000 hasta el 2013 (ambos incluidos), agregados en dos periodos temporales (2000-2007 y 2008-2013).

Se han estudiado los movimientos residenciales de personas, y no de núcleos familiares, por dos motivos, uno de carácter teórico y otro de carácter metodológico. En primer lugar, la creciente transformación del ciclo del hogar tradicional (diversificación de los modos de convivencia, separación y recomposición familiares, diversidad entre grupos sociales y étnicos, etc.) pone en entredicho la concepción clásica del hogar como unidad de análisis de la movilidad residencial, al suponer precisamente un fenómeno que a menudo se asocia con una transformación (disolución, unión, etc.) del hogar o de los hogares originarios. Por ello, algunos autores consideran conveniente poner el foco en los recorridos individuales sin tratar de pensar en las carreras familiares, lo que supone lidiar con mayores problemas metodológicos (Duncan y Hill, 1985 en Mulder, 1993). En segundo lugar, y relacionado con estas dificultades, no es posible contar con información estadística sobre la movilidad de los hogares con el nivel de desagregación espacial y temporal y por un periodo tan largo de tiempo requeridos en este análisis. 
La fuente empleada en el estudio es el Padrón Municipal de Habitantes. A fin de medir la movilidad residencial, se han considerado, a partir de las altas y bajas del Padrón Municipal de Habitantes, todos los desplazamientos que implicaban un cambio de vivienda con origen o destino en alguno de los 128 barrios del municipio de Madrid, independientemente de si estos desplazamientos conllevan cambios en otras esferas además de la residencial como consecuencia de su distancia geográfica? .

Para ello, se solicitó una explotación específica que ha permitido ajustar la información espacial y temporalmente a los objetivos de la investigación. A pesar de que la información sociodemográfica contenida en los registros padronales es limitada ${ }^{10}$, la posibilidad de su desagregación permite realizar una aproximación única, no posibilitada por otras fuentes estadísticas.

Para el análisis a escala de barrio, se han realizado las operaciones necesarias para poder distinguir entre los movimientos de entrada y salida de cada uno de los barrios de aquellos que se producen en el interior de los mismos. A partir de ellos, se han calculado diferentes indicadores que pretenden medir la intensidad y la evolución de la movilidad residencial:

- Tasa anual de movilidad (TAM). Indicador bruto de intensidad que relaciona todos los movimientos residenciales que afectan a cada unidad territorial con la población media de la misma:

$$
\text { TAM }=\frac{\left(\text { Entradas }^{t, t+n}+\text { Salidas }^{t, t+n}+\text { Movimientos internos }^{t, t+n}\right) / n}{\frac{1}{2} *\left(\text { Pob }^{t}+\text { Pob }^{t+n}\right)} * 1000
$$

- Indice de atracción residencial (IAR). Mide globalmente la intensidad de las nuevas entradas en el barrio, independientemente del origen de las mismas:

$$
\mathrm{IAR}=\frac{(\text { Entradas desde otros barrios }}{\left.{ }^{t, t+n}+\text { Entradas desde fuera del municipio }^{t, t+n}\right) / n} * 1000
$$

9. La distinción entre las migraciones y los movimientos residenciales desde una interpretación sociológica es que las primeras suponen una modificación del espacio de vida (Courgeau, 1988; Susino, 2003) y las segundas, no (o no necesariamente). Sin embargo, su estudio está sujeto a administraciones territoriales que no aseguran la concordancia entre definición teórica y análisis empírico, y, de hecho, los conceptos son a menudo mezclados, como en el caso de las intraurban migration o las mobilités résidentielles en el estudio de las migraciones interiores francesas. Si bien es cierto que los significados y las consecuencias en la vida de quienes los protagonizan son enormemente distantes, a efectos de analizar la relación entre la movilidad y los cambios en la composición social del espacio urbano, se ha considerado contemplar la totalidad de los desplazamientos residenciales.

10. Las variables sociodemográficas que recoge el Padrón Municipal de Habitantes son: edad, sexo, lugar de nacimiento, nacionalidad y nivel de estudios. 
- Tasa de salidas residenciales (TSR). Relaciona el número total de los desplazamientos residenciales que suponen una salida (independientemente del destino del movimiento) con la población media del mismo:

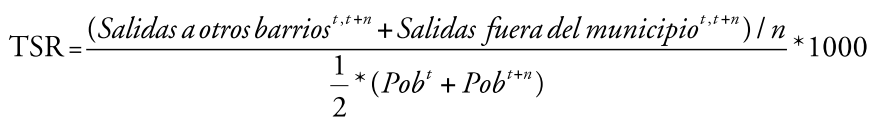

- Tasa de movilidad intrabarrio (TMI). A fin de medir la intensidad de los movimientos que tienen como origen y destino la misma unidad territorial:

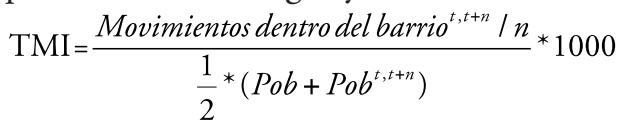

A partir de los últimos tres indicadores ${ }^{11}$, se ha realizado un análisis de conglomerados mediante el método K-medias para cada uno de los periodos analizados ${ }^{12}$.

En la segunda etapa del análisis, se han elaborado índices de variación de la composición sociodemográfica a partir de las variables padronales que miden la variación de la población empadronada al inicio y al final de cada uno de los periodos considerados. A fin de analizar la relación entre la movilidad y las transformaciones en la composición social de los barrios madrileños, se ha analizado su relación estadística con la tasa de movilidad. Para ello, se ha realizado una comparación de medias de los indicadores de variación de cada tipo de barrio en función de su movilidad y se han analizado los coeficientes de correlación de Pearson obtenidos del cruce de los indicadores de variación con la tasa de movilidad. Aunque no se han comentado en detalle, se han añadido las rectas de regresión y los $\mathrm{R}^{2}$ que añaden capacidad explicativa de la tasa de movilidad en las variaciones sociodemográficas. Estos cruces permiten ver en qué medida las variaciones en la composición sociodemográfica de los barrios madrileños se deben a los movimientos residenciales.

También se ha introducido información de las prácticas de movilidad residencial a fin de analizar si los cambios producidos en uno y otro periodo se debían a una modificación de la estructura poblacional, a la modificación de las prácticas de ciertos grupos sociales o a la modificación de su inserción territorial.

11. Conviene aclarar que la Tasa de Migración Neta es un mal indicador de intensidad respecto a los objetivos planteados. Al considerar como numerador de la ecuación el saldo migratorio, solo sería un indicador efectivo en aquellos casos en que las entradas y las salidas fuesen muy diferentes en intensidad. Los barrios con alto reemplazo poblacional presentarían un saldo migratorio próximo a 0 y no serían identificados como zonas de alta movilidad o de reemplazo poblacional.

12. Como toda tasa, son de carácter anual, por lo que se han calculado a partir del promedio anual de movimientos de cada periodo entre la población media del mismo. Para eliminar los efectos de la incorporación del ejercicio censal de 2001 y asegurar la comparabilidad entre periodos temporales en cuanto a las características de la fuente, se ha excluido dicho año en todos los análisis referentes al periodo 2000-2007. 


\section{La interpretación territorial a partir de las prácticas socioespaciales}

La caracterización de los barrios madrileños en función de la movilidad residencial evidencia una estabilidad espacial generalizada de las categorías resultantes ${ }^{13}$. Como se aprecia en los mapas 1 y 2 , los barrios han sido clasificados como sigue:

1. Barrios de alta movilidad, que parecen encontrarse en «estado de ebullición», pues en ellos establecen su residencia personas provenientes de fuera de los mismos, a la vez que son abandonados en intensidad casi pareja y con una alta movilidad interna.

2. Barrios de movilidad media, tanto en entradas residenciales como en salidas y en la movilidad intrabarrio.

3. Barrios de movilidad baja, caracterizados por la inmovilidad física y una estabilidad, por tanto, de los agentes sociales que los ocupan.

4. Barrios receptores, que presentan tasas altas de entrada pero tasas reducidas de salida y tasas medias o bajas de movilidad intrabarrios.

Resulta ahora necesario explorar cuál es el efecto de estas distintas intensidades de la movilidad residencial en la transformación de su composición social a partir de la relación entre la intensidad de la movilidad con indicadores de transformación sociodemográfica que las variables padronales permiten construir.

\section{Transformaciones en la composición por edad}

La movilidad residencial se asocia con un rejuvenecimiento de la composición social de los barrios madrileños, especialmente durante el primer periodo analizado en el que se asocia a una reducción en la edad media y un aumento del índice de juventud en aquellos barrios más afectados por los cambios residenciales (tabla 1). Esta asociación manifiesta que la edad media se ha reducido más en aquellos barrios con alta movilidad que en los que tuvieron una intensidad media o baja, destacando el caso de los barrios receptores, en los que la edad media disminuyó en casi cinco años como promedio durante el primer periodo (tabla 2). Si bien su efecto en la disminución de la edad media y en la proporción de mayores continúa en el segundo periodo, esta ya no se relaciona con un aumento de los jóvenes, sino con un aumento de la proporción de adultos, con lo que desaparece su capacidad explicativa en las variaciones de la proporción de jóvenes ( $27 \%$ de la varianza explicada en el primer periodo) (gráficos 1 y 2).

13. Se han realizado pruebas en el análisis de conglomerados modificando el número de categorías resultantes. Tras la realización de las diferentes pruebas, se ha considerado que la agrupación en cuatro conglomerados es la que mayor solidez estadística presenta y la que más se ajusta a los objetivos teóricos perseguidos. Para mayor información sobre la clasificación resultante, ver la tabla 1 del anexo. 
Mapas 1 y 2. Clasificación de los barrios madrileños en función del tipo de movilidad residencial resultante del análisis de conglomerados por el método K-medias

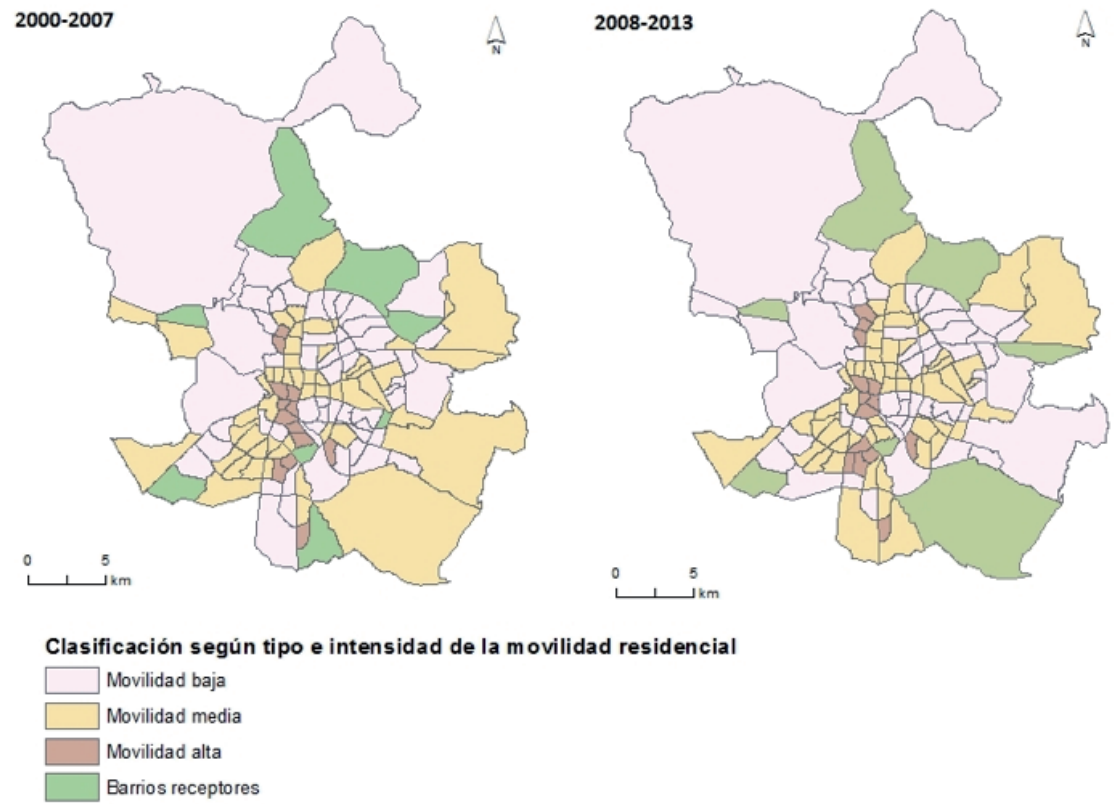

Fuente: elaboración propia. Padrón Municipal de Habitantes.

Tabla 1. Coeficientes de correlación de Pearson entre la tasa de movilidad y los indicadores de variación de la estructura por edad ${ }^{14}$. Periodos 2000-2007 y 2008-2013

\begin{tabular}{lcc}
\hline & $\begin{array}{c}\text { Coef. correlación } \\
\text { 2000-2007 }\end{array}$ & $\begin{array}{c}\text { Coef. correlación } \\
\mathbf{2 0 0 8 - 2 0 1 3}\end{array}$ \\
\hline Variación de la edad media & $-0,713^{\star *}$ & $-0,390^{\star \star}$ \\
Variación del índice de infancia & $0,273^{\star *}$ & $0,232^{\star \star}$ \\
Variación del índice de juventud & $0,527^{\star \star}$ & $-0,224^{\star}$ \\
Variación del índice de adultos & $0,185^{\star}$ & $0,567^{\star *}$ \\
Variación del índice de envejecimiento & $-0,698^{\star *}$ & $-0,561^{\star *}$ \\
\hline
\end{tabular}

* La correlación es significativa en el nivel 0,05 (2 colas).

** La correlación es significativa en el nivel 0,01 (2 colas).

Fuente: elaboración propia. Padrón Municipal de Habitantes.

14. El primero de ellos consiste en la variación de la edad media, útil para detectar procesos de envejecimiento y de rejuvenecimiento generales. Los otros cuatro indicadores hacen referencia a la variación, en puntos porcentuales, de la proporción que suponen estos cuatro grandes grupos de edad en cada uno de los 128 barrios del municipio de Madrid. Índice de infancia: proporción de personas menores de 15 años; indice de juventud: proporción de personas entre 15 y 34 años; indice de adultos: proporción de personas entre 35 y 64 años; indice de envejecimiento: proporción de personas de 65 años y más. 
Tabla 2. Valor medio y desviación típica de los indicadores de variación de la estructura de edad en cada tipo de barrio según movilidad. Periodos 2000-2007 y 2008-2013 ${ }^{15}$

\begin{tabular}{|c|c|c|c|c|c|c|c|c|}
\hline \multirow{2}{*}{ Tipo de movilidad } & \multicolumn{2}{|c|}{ Baja } & \multicolumn{2}{|c|}{ Media } & \multicolumn{2}{|c|}{ Alta } & \multicolumn{2}{|c|}{ Receptores } \\
\hline & Media & SD & Media & SD & Media & SD & Media & SD \\
\hline \multicolumn{9}{|l|}{ Periodo 2000-2007 } \\
\hline Variación en la Edad Media & 1,8 & 1,4 & $-0,5$ & 1,4 & $-2,4$ & 1,1 & $-4,8$ & 7,0 \\
\hline Variación Índice de Infancia & 0,1 & 1,9 & 0,8 & 1,6 & 0,3 & 1,3 & 5,6 & 6,3 \\
\hline Variación Índice de Juventud & $-4,8$ & 3,6 & $-1,3$ & 2,8 & 2,8 & 1,7 & $-3,8$ & 9,5 \\
\hline Variación Índice de Adultos & 2,9 & 2,9 & 2,7 & 1,3 & 3,2 & 1,1 & 6,8 & 6,0 \\
\hline Variación Índice de Envejecimiento & 1,9 & 2,8 & $-2,2$ & 3,0 & $-6,3$ & 1,8 & $-8,6$ & 12,0 \\
\hline \multicolumn{9}{|l|}{ Periodo 2008-2013 } \\
\hline Variación en la Edad Media & 2,1 & 0,9 & 1,4 & 0,8 & 1,0 & 0,4 & 0,1 & 2,8 \\
\hline Variación Índice de Infancia & $-0,1$ & 1,5 & 0,4 & 1,2 & 0,4 & 0,8 & 2,2 & 3,9 \\
\hline Variación Índice de Juventud & $-4,1$ & 2,4 & $-4,5$ & 2,7 & $-4,7$ & 1,2 & $-8,2$ & 3,1 \\
\hline Variación Índice de Adultos & 1,0 & 2,2 & 2,8 & 2,0 & 4,4 & 0,9 & 7,3 & 4,8 \\
\hline Variación Índice de Envejecimiento & 3,2 & 2,4 & 1,3 & 1,4 & $-0,2$ & 0,8 & $-1,3$ & 3,2 \\
\hline
\end{tabular}

Fuente: elaboración propia. Padrón Municipal de Habitantes.

Gráficos 1 y 2. Diagramas de dispersión simple en la tasa de movilidad y variación en el índice de juventud en los barrios de Madrid. Periodos 2000-2007 y 2008-2013

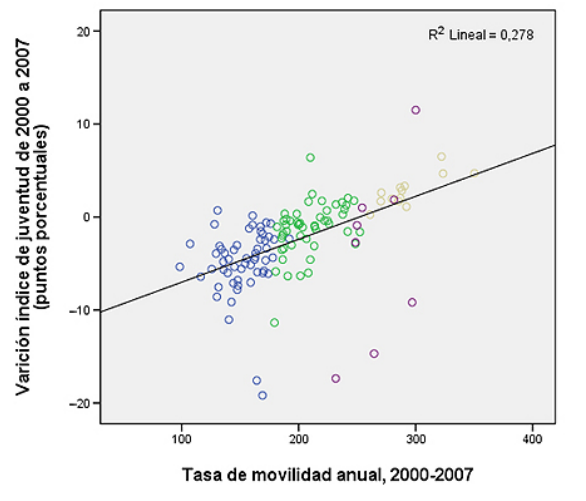

Movilidad baja

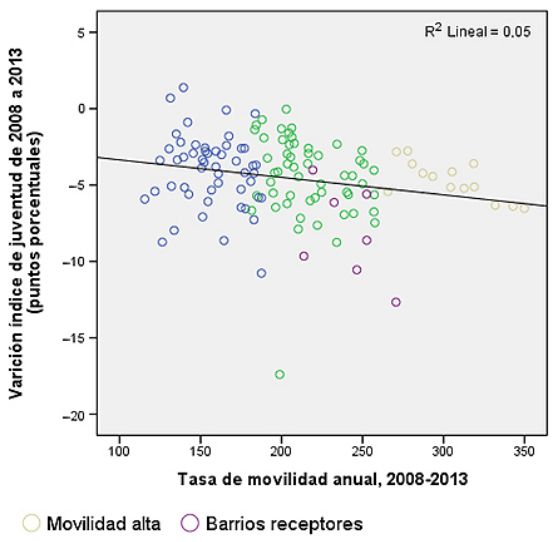

Movilidad alta $\bigcirc$ Barrios receptores

Fuente: elaboración propia. Padrón Municipal de Habitantes.

15. Se ha realizado una comparación de medias para todos aquellos indicadores que se relacionan con la movilidad: indicadores de variación de la estructura por edad; variación de la proporción de nacidos en el extranjero, e indicadores de variación de nivel educativo. La comparación de medias se ha realizado mediante el ANOVA de un factor y el estadístico F, para un nivel de significación de 0,05 , ha concluido que la diferencia de medias de al menos uno de los grupos es estadísticamente significativa para todos los indicadores en los dos periodos analizados, salvo en uno de ellos, indicado más adelante. 
Gráfico 3. Proporción de cada grupo de edad sobre el total de movimientos en el municipio de Madrid en los periodos 2000-2007 y 2008-2013

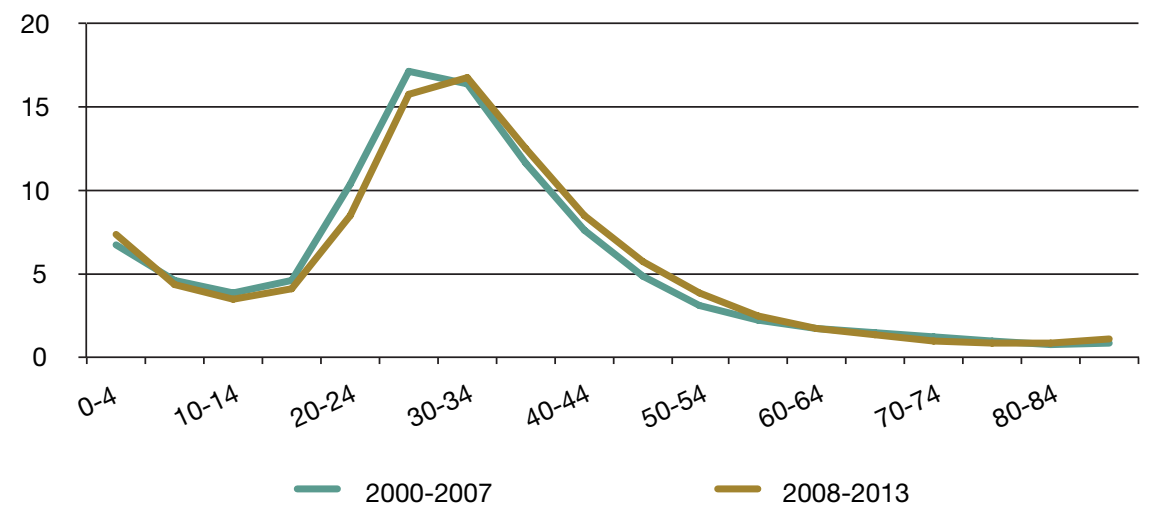

Fuente: elaboración propia. Padrón Municipal de Habitantes.

La diferencia en el efecto de la movilidad residencial en los procesos de rejuvenecimiento puede deberse, en parte, a una menor participación de los jóvenes en los cambios residenciales, por una reducción de su peso sobre la población del municipio (pasan de suponer el 30\% del total poblacional en el año 2000 al 23\% en el 2014), y no a una modificación de sus prácticas residenciales. Así, en el segundo periodo, se produce un aumento en la edad de los agentes sociales que protagonizan los cambios residenciales (gráfico 3), debido a una modificación de la composición por edad del espacio social y no a una reducción de los cambios de residencia entre los jóvenes. Por el contrario, las tasas específicas de movilidad intramunicipal ${ }^{16}$ muestran una intensificación de los cambios residenciales en todos los grupos de edad menores de 65 años, especialmente acuciada en los grupos de 20 a 39 años (gráfico 4).

Como se decía, esta menor participación de los jóvenes en el total de cambios de vivienda en el segundo periodo podría explicar en parte la desaparición del efecto de la movilidad residencial en el rejuvenecimiento de los barrios madrileños. Sin embargo, merece la pena destacar que la asociación entre la movilidad y una mayor presencia de jóvenes no desaparece en el segundo periodo. Si, en lugar de considerar la variación del índice de juventud, se considera directamente la proporción de jóvenes en la fecha de finalización de cada uno de los periodos analizados, se observa que es precisamente en aquellos barrios con mayor movilidad en los que hay una mayor proporción de jóvenes (grá-

16. Tasas específicas por edad: «relación existente entre las migraciones realizadas por los migrantes de una edad determinada [...] con la población media de dicha edad» (Vinuesa et al., 1994: 157). Solo pueden ser obtenidas para los movimientos internos y para las salidas residenciales o emigraciones, por la necesidad de conocer para su cálculo la población de referencia (Martín y Rodríguez, 2007). 
Gráfico 4. Tasas específicas de movilidad en el municipio de Madrid en los periodos 20002007 y $2008-2013$

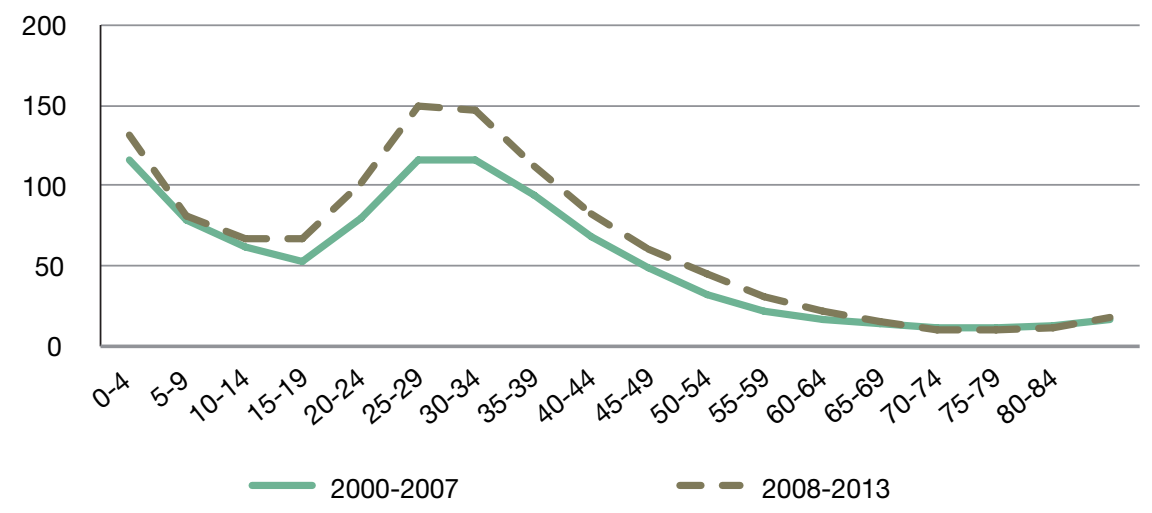

Fuente: elaboración propia. Padrón Municipal de Habitantes.

Gráficos 5 y 6 . Diagramas de dispersión simple en la tasa de movilidad e índice de juventud en los barrios de Madrid. Periodos 2000-2007 y 2008-2013

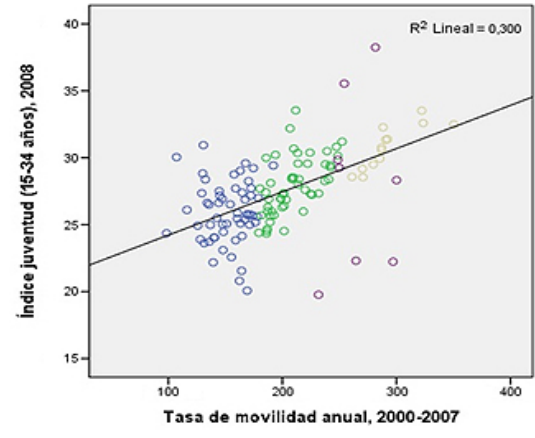

Movilidad baja

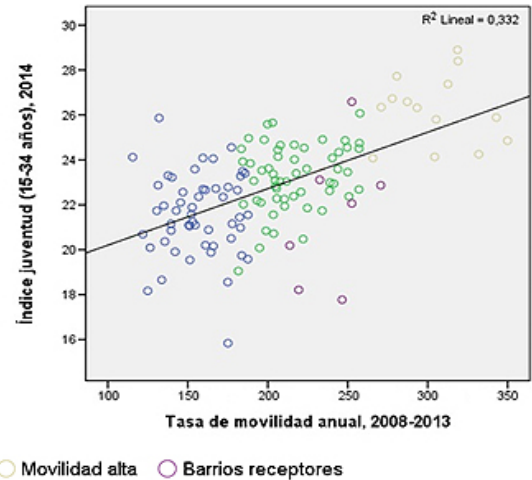

Movilidad alta $\bigcirc$ Barrios receptores

Fuente: elaboración propia. Padrón Municipal de Habitantes.

ficos 5 y 6). Esto parece indicar que, en el segundo periodo, los jóvenes que cambian de vivienda se instalan en barrios que ya contaban con una elevada proporción de ellos antes de su llegada o en barrios que son abandonados en la misma intensidad por otros jóvenes, por lo que su desplazamiento no estaría provocando efectos en el peso proporcional de su grupo de edad. Por tanto, las diferencias entre los dos periodos analizados no solo se explicarían por una modificación en la edad de los agentes sociales que realizan los cambios de vivienda, sino también por una modificación socioespacial de las prácticas residenciales de los jóvenes. 
Cabe mencionar los valores que presentan los indicadores de variación por edad en los barrios receptores. En estos barrios, ha aumentado la proporción de menores de 15 años y de adultos significativamente, mientras se ha reducido la de jóvenes y mayores. Estas variaciones están muy posiblemente indicando una apropiación de los mismos por hogares con presencia de niños o en los años muy anteriormente inmediatos a su nacimiento.

\section{Variación según país de nacimiento ${ }^{17}$}

La importante llegada de personas desde el extranjero durante los últimos años del siglo pasado y los primeros del actual ha conllevado, sin duda, una de las transformaciones más significativas en el espacio social de nuestro ámbito de los últimos tiempos. Estas llegadas han estado esencialmente protagonizadas por lo que se conoce como «inmigración laboral», concepto que realmente no responde a la dimensión productiva en las razones de la migración, sino que pretende aglutinar a todas aquellas personas extranjeras desposeídas de capital o que ocupan posiciones sociales desaventajadas. Si bien es cierto que las condiciones residenciales de la población inmigrante varían en función de la nacionalidad (Cortés et al., 2004) o de la fase en el proceso de integración, la condición de «inmigrante económico» se asocia a una desposesión de capitales simbólico (que se manifiesta a través de prácticas discriminatorias) y social, que, a menudo, se vincula con falta de capital económico. Como consecuencia, los extranjeros suelen contar con peores posiciones residenciales que la población autóctona (Colectivo IOÉ, 2005; Hernández y López, 2013; Andújar y Feria, 2015), por lo que es presumible que las prácticas sociespaciales que desarrollan sean también diferenciadas de las de aquellos que cuentan con mejores posiciones sociales.

Las personas nacidas en el extranjero en el municipio de Madrid pasaron de suponer cerca de un 5\% de la población en el año 2000 a casi un $20 \%$ en 2008 , proporción que se mantiene prácticamente estable hasta el 1 de enero de 2014. Cabe preguntarse: ¿qué efectos ha conllevado dicha circunstancia en los barrios madrileños?; ‘ha supuesto transformaciones en todos ellos?; una vez establecidos en el municipio, ¿las prácticas de movilidad residencial tienden a equilibrar las diferencias de localización o, por el contrario, tienden a agudizarlas?

La fuerza de las inmigraciones internacionales en el primer periodo conlleva una asociación lineal positiva entre la movilidad y la variación de nacidos en el extranjero, con un $42,6 \%$ del porcentaje de la varianza de esta última explicado por la tasa de movilidad (gráficos 7 y 8). A pesar de esta tendencia general,

17. Para analizar a la población inmigrante, se ha considerado la variable país de nacimiento en lugar de nacionalidad. La nacionalidad se refiere al estatus legal en un proceso en el que resulta muy difícil establecer la línea acerca de cuándo un inmigrante deja de serlo (no solo desde el punto de vista legal, sino también social y simbólico), y si bien esta puede producirse en una fase del proceso migratorio ya avanzado, su consecución no implica la desaparición de prácticas sociales ni residenciales diferenciadas con respecto a la población autóctona. 
Tabla 3. Valores medios y desviación típica de la variación del porcentaje de extranjeros por tipos de barrio según su movilidad. Periodos 2000-2007 y 2008-2013

\begin{tabular}{|c|c|c|c|c|c|c|c|c|}
\hline \multirow[b]{2}{*}{ Tipo de movilidad } & \multicolumn{2}{|c|}{ Baja } & \multicolumn{2}{|c|}{ Media } & \multicolumn{2}{|c|}{ Alta } & \multicolumn{2}{|c|}{ Receptores } \\
\hline & Media & SD & Media & SD & Media & SD & Media & SD \\
\hline $\begin{array}{l}\text { Variación en la proporción } \\
\text { de extranjeros } 2000-2007\end{array}$ & 9,8 & 4,0 & 15,8 & 4,9 & 23,1 & 7,7 & 8,7 & 4,5 \\
\hline $\begin{array}{l}\text { Variación en la proporción } \\
\text { de extranjeros } 2008-2014\end{array}$ & $-0,3$ & 1,2 & 0,0 & 1,2 & 0,6 & 1,7 & $-1,5$ & 2,4 \\
\hline
\end{tabular}

Fuente: elaboración propia. Padrón Municipal de Habitantes.

merece destacar el comportamiento dispar de algunos barrios. En primer lugar, despuntan algunos en los que la variación de población nacida en el extranjero ha sido más pronunciada que su tasa de movilidad. San Cristóbal, Almendrales, San Diego y Embajadores son algunos de ellos. Se trata de barrios en los que tradicionalmente han residido clases populares y que presentan los mayores porcentajes de población nacida en el extranjero en la fecha de finalización de los dos periodos analizados. En el extremo opuesto, se encuentran los barrios receptores, que cuentan con altas tasas de movilidad (fundamentalmente compuestas por entradas) y, contrariamente, son en los que menos aumenta o incluso disminuye este grupo (tabla 3 y gráficos 7 y 8).

La capacidad explicativa de la tasa de movilidad en la variación de nacidos en el extranjero desaparece en el segundo periodo de análisis.

Estas diferencias entre periodos, ¿responden a un cambio en las prácticas residenciales de la población extranjera entre el primer y el segundo periodos analizados? ¿Es una participación similar en los cambios residenciales entre la

Gráficos 7 y 8 . Diagramas de dispersión simple en la tasa de movilidad y variación en la proporción de población nacida en el extranjero. Periodos 2000-2007 y 2008-2013

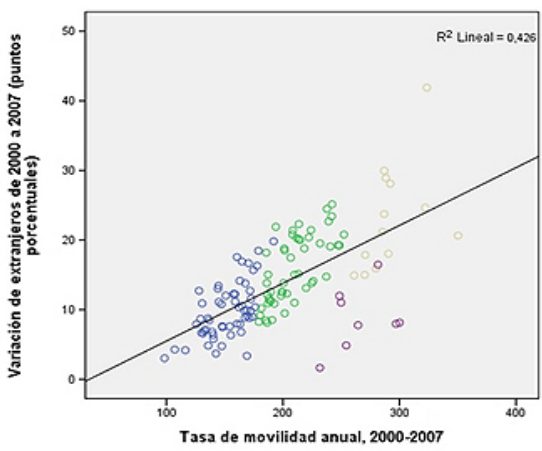

Movilidad baja

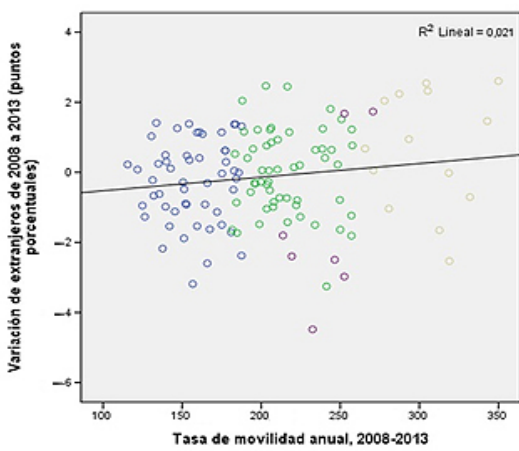

Movilidad alta $\bigcirc$ Barrios receptores

Fuente: elaboración propia. Padrón Municipal de Habitantes. 
Tabla 4. Índice sintético de movilidad intramunicipal por país de nacimiento y periodo temporal

\begin{tabular}{lcccc}
\hline & & España & Extranjero & Total \\
\hline ISM intramunicipal & $2000-2007$ & 3,3 & 14,3 & 4,9 \\
& $2008-2013$ & 3,6 & 13,5 & 5,8 \\
\hline
\end{tabular}

Fuente: elaboración propia. Padrón Municipal de Habitantes.

población nacida en España y la nacida en el extranjero la que reduce el efecto de la movilidad residencial en el segundo periodo analizado? La respuesta a estas preguntas es negativa. Si atendemos al índice sintético de movilidad intramunicipal ${ }^{18}$ (tabla 4), observamos que, a pesar de una reducción entre los nacidos en el extranjero y un leve aumento entre los nacidos en España, las prácticas residenciales que implican un cambio de vivienda siguen siendo muy dispares entre unos y otros: la movilidad entre los nacidos fuera sigue siendo casi cuatro veces superior a la de los españoles entre 2008 y 2013.

La elevada movilidad que sigue protagonizando la población nacida en el extranjero una vez asentada en el municipio indica que la ausencia de efectos que la movilidad conlleva en la variación de este grupo no solo se explica a partir de la disminución de nuevas llegadas al municipio. Si bien esta supone una explicación parcial, su alta rotación residencial podría seguir implicando transformaciones en los barrios madrileños. De hecho, si se considera el porcentaje de población nacida fuera (tomada como fotografía del final de cada uno de los periodos), la asociación entre variables aumenta, y de manera muy significativa en el segundo periodo, con un coeficiente de correlación de $0,862^{19}$ y un $\mathrm{R}^{2}$ del $74 \%$ (gráficos 9 y 10). Así, una mayor intensidad de la movilidad residencial se relaciona con una mayor proporción de nacidos fuera, pero sin un aumento de la misma en el segundo periodo. ¿Qué indica el hecho de que haya una asociación tan fuerte entre la movilidad y la presencia de nacidos en el extranjero y, sin embargo, no suponga un aumento de este grupo? La explicación se encuentra en la relación con el espacio que estas prácticas residenciales conllevan. Los datos estarían mostrando que, en el segundo periodo analizado, la población nacida en el extranjero se asienta en barrios en los que ya existe una proporción elevada de extranjeros o en los que también hay salidas de este colectivo, por lo que no genera trasformaciones con su llegada.

En ambos periodos, los barrios receptores vuelven a desmarcarse de esta pauta, con reducciones o mantenimiento de este grupo, lo que evidencia su ausencia entre quienes han llegado a vivir a estos barrios.

18. El ISM es el sumatorio de las tasas específicas de movilidad y expresa el número de cambios de vivienda que realizaría una persona perteneciente a una generación ficticia si, a lo largo de su vida, pasara por todas las tasas específicas obtenidas. De esta manera se elimina el efecto de la estructura por edad revelando la intensidad de la movilidad sin interferencias (Martín y Rodríguez, 2007).

19. La correlación es significativa en el nivel 0,01 ( 2 colas). 
Gráficos 9 y 10. Diagramas de dispersión simple en la tasa de movilidad y porcentaje de nacidos en el extranjero en los barrios de Madrid. Periodos 2000-2007 y 2008-2013. Marcas por tipo de movilidad
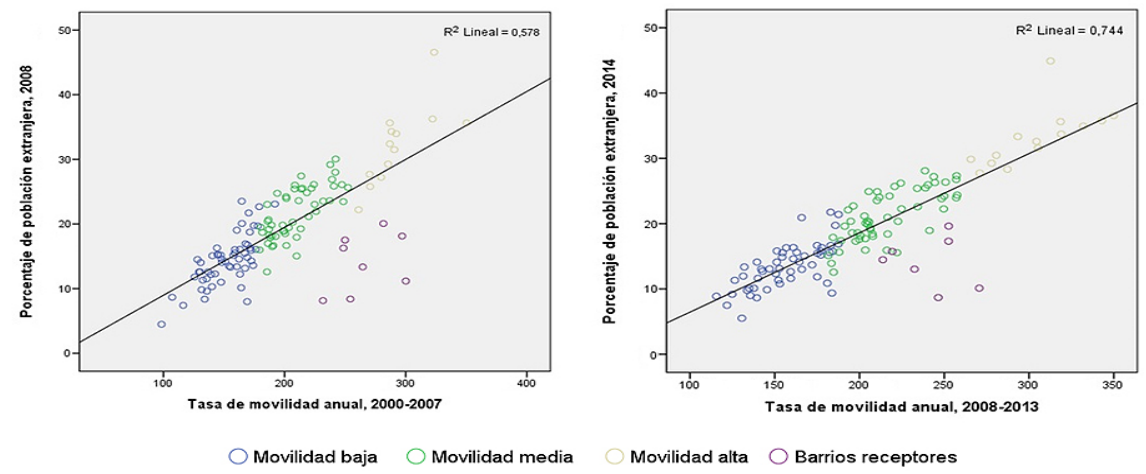

Fuente: elaboración propia. Padrón Municipal de Habitantes.

\section{Variación según el nivel de estudios}

La variable padronal nivel de estudios sirve como proxy para analizar el capital cultural y la posición social de los agentes sociales. Antes de entrar en el análisis, conviene señalar que es esperable que los barrios con mayores entradas de población se asocien a grados formativos más elevados por dos cuestiones:

- En primer lugar, el cambio social producido en las últimas décadas, compuesto, entre otros, por un aumento de los niveles formativos, conlleva que los jóvenes (con mayor movilidad residencial) cuenten con un grado académico más elevado que las personas de más edad, más estables residencialmente.

- En segundo lugar, por una distorsión de la fuente debida a la falta de actualización del nivel de estudios de aquellos que no cambian de vivienda, por lo que resulta muy probable que se produzca una subrepresentación del grado académico entre ellos.

Sin embargo, una aproximación al análisis de las transformaciones relacionadas con el nivel de estudios puede proporcionar alguna información relevante.

Atendiendo a las categorías más extremas de esta variable, se aprecia una relación general entre la intensidad de la movilidad y una reducción de la proporción de personas sin estudios. Como muestran los gráficos 11 y 12, las rectas de regresión indican que, si bien la movilidad se encuentra entre las explicaciones de la disminución de la población sin estudios, la capacidad explicativa de esta es algo reducida (un 20,6\% de la varianza explicada por la tasa de movilidad en el primer periodo y un $31 \%$ en el segundo). 
Tabla 5. Indicadores de variación del nivel de estudios de los mayores de 25 años por categorías de barrios en función de su movilidad (valores medios y desviación típica). Periodos $2000-2007$ y $2008-2013^{20}$

\begin{tabular}{|c|c|c|c|c|c|c|c|c|}
\hline \multirow{2}{*}{ Tipo Movilidad } & \multicolumn{2}{|c|}{ Baja } & \multicolumn{2}{|c|}{ Media } & \multicolumn{2}{|c|}{ Alta } & \multicolumn{2}{|c|}{ Receptores } \\
\hline & Media & SD & Media & SD & Media & SD & Media & SD \\
\hline \multicolumn{9}{|l|}{ Periodo 2000-2007 } \\
\hline $\begin{array}{l}\text { Variación de población sin } \\
\text { estudios }\end{array}$ & $-8,2$ & 3,8 & $-10,9$ & 5,3 & $-12,5$ & 1,6 & $-25,6$ & 19,5 \\
\hline $\begin{array}{l}\text { Variación de población con } \\
\text { primaria o secundaria }\end{array}$ & 3,3 & 3,0 & 4,8 & 3,6 & 5,9 & 4,1 & 1,1 & 6,1 \\
\hline $\begin{array}{l}\text { Variación de población con } \\
\text { estudios medios }\end{array}$ & 4,3 & 1,3 & 4,0 & 1,5 & 3,1 & 1,2 & 6,1 & 7,5 \\
\hline $\begin{array}{l}\text { Variación de población con } \\
\text { estudios superiores }\end{array}$ & 2,0 & 2,0 & 3,6 & 3,6 & 4,7 & 3,3 & 19,7 & 11,7 \\
\hline \multicolumn{9}{|l|}{ Periodo 2008-2013 } \\
\hline $\begin{array}{l}\text { Variación de población sin } \\
\text { estudios }\end{array}$ & $-3,7$ & 1,6 & $-4,9$ & 1,4 & $-7,0$ & 1,5 & $-8,0$ & 5,9 \\
\hline $\begin{array}{l}\text { Variación de población con } \\
\text { primaria o secundaria }\end{array}$ & $-1,0$ & 1,5 & $-0,6$ & 1,9 & 0,8 & 2,4 & $-2,1$ & 1,8 \\
\hline $\begin{array}{l}\text { Variación de población con } \\
\text { estudios medios }\end{array}$ & $-2,6$ & 2,5 & $-1,4$ & 2,3 & $-0,2$ & 1,0 & $-0,2$ & 3,5 \\
\hline $\begin{array}{l}\text { Variación de población con } \\
\text { estudios superiores }\end{array}$ & 7,5 & 2,7 & 7,1 & 3,3 & 6,5 & 4,1 & 10,3 & 3,0 \\
\hline
\end{tabular}

Fuente: elaboración propia. Padrón Municipal de Habitantes.

La otra categoría más relevante como indicador de transformación de la composición social es la proporción de personas con estudios superiores. Como se aprecia en los gráficos 13 y 14, la asociación y el signo entre variables cambian de un periodo a otro. Entre 2000 y 2007, hay una asociación positiva indicando que, en aquellos barrios con mayor tasa de movilidad, se ha producido un aumento más elevado del porcentaje de personas con estudios superiores. En el segundo periodo, la intensidad de la movilidad no conlleva efectos significativos respecto a la proporción de población con estudios superiores, justo a la inversa que la que habría podido predecirse por los problemas de actualización de la fuente señalados anteriormente. Esta falta de asociación en el segundo periodo parece explicarse más por la relación con el espacio que por las prácticas residenciales. Si bien las tasas de movilidad por nivel educativo muestran una reducción de los cambios residenciales entre aquellos más formados, su participación en el total de cambios residenciales es mayor debido al aumento de la población con estudios superiores. Por

20. En la comparación de medias mediante el ANOVA de un factor, y el estadístico F, para un nivel de significación de 0,05 , ha concluido que la diferencia de medias de al menos uno de los grupos no es estadísticamente significativa para el indicador «Variación de población con estudios superiores» en el periodo 2008-2013. 
Gráficos 11 y 12. Diagramas de dispersión simple en la tasa de movilidad y variación de la proporción de población mayor de 25 años sin estudios en los barrios de Madrid. Periodos 2000-2007 y 2008-2013

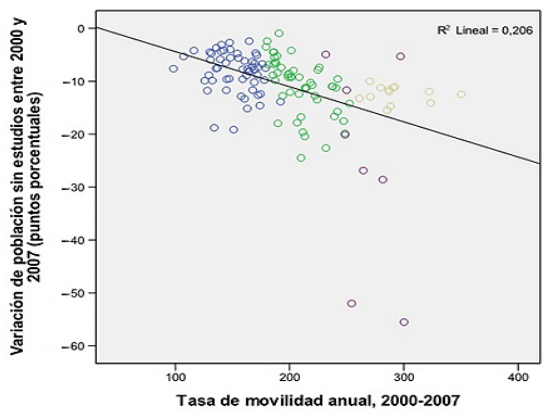

Movilidad baja $\bigcirc$ Movilidad media

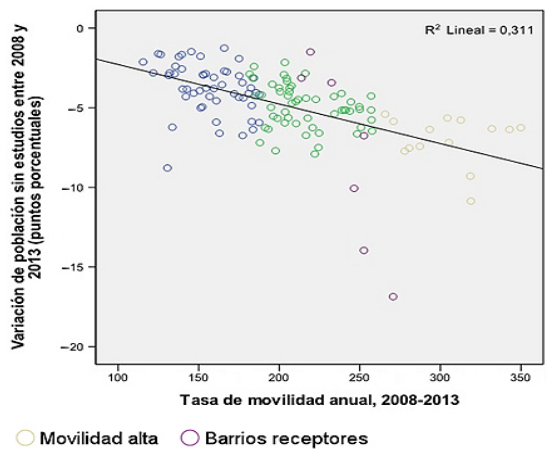

Fuente: elaboración propia. Padrón Municipal de Habitantes.

Gráficos 13 y 14. Diagramas de dispersión simple en la tasa de movilidad y variación de la proporción de población mayor de 25 años con estudios superiores en los barrios de Madrid. Periodos 2000-2007 y 2008-2013
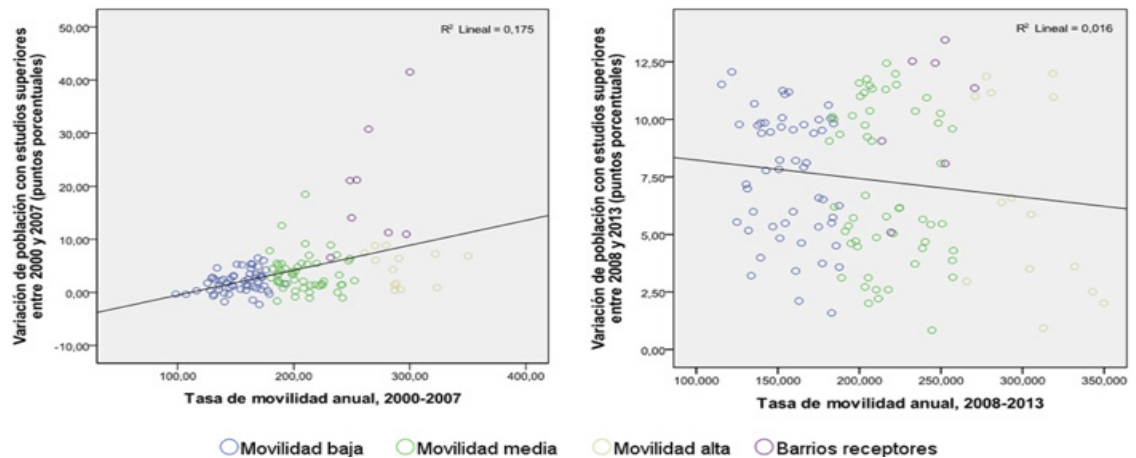

Fuente: elaboración propia. Padrón Municipal de Habitantes.

tanto, la explicación parece encontrarse de nuevo en la dimensión socioespacial, lo que provoca que su movilidad no conlleve transformaciones en los barrios a los que implica.

Volviendo a las tasas por nivel educativo, cabe señalar que se aprecia una intensificación de los cambios residenciales entre aquellas personas sin estudios y una reducción en todas las demás categorías. Esto parece indicar un aumento de la movilidad entre los peor posicionados, lo que podría relacionarse con situaciones de precaridad residencial (gráfico 15).

En cualquier caso, en este segundo periodo destaca la gran dispersión interna en cuanto a la variación de población con estudios superiores en los barrios 
Gráfico 15. Tasas de movilidad por grupos según el nivel educativo (mayores de 25 años)

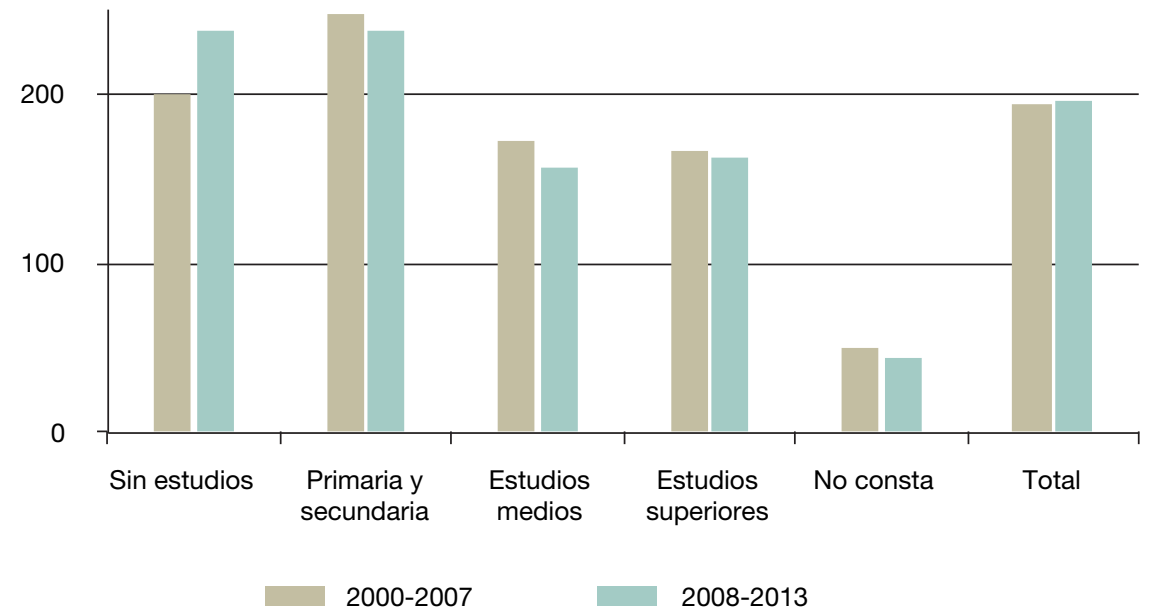

Fuente: elaboración propia. Padrón Municipal de Habitantes.

de baja, media y alta movilidad. Poniendo la atención en aquellos de mayor movilidad, pueden distinguirse dos grupos que se alejan de la media. Uno de ellos, por contar con una de las tasas más elevadas de movilidad y de los menores incrementos de la población con estudios superiores. Entre ellos, destacan San Cristóbal, Almendrales, Moscardó, San Diego, Padrolongo y Zofio, que, de nuevo, se identifican con zonas tradicionalmente populares y que en el primer periodo ya presentaban una fuerte intensidad de movilidad residencial y un incremento muy reducido de la proporción de población más formada. El otro grupo identificable es el compuesto por Embajadores Cortes, Justicia, Universidad y Sol, del distrito Centro, con elevadas tasas de movilidad acompañadas de incrementos de la proporción de población con estudios superiores.

Los barrios receptores destacan muy significativamente, con un aumento promedio de la proporción de personas con estudios superiores de casi 18 puntos porcentuales entre 2000 y 2007, y también con el mayor incremento medio en el segundo periodo (tabla 5). Esta categoría de barrios, por tanto, destaca por el crecimiento del nivel educativo, especialmente a causa de sus incrementos en la proporción de población con estudios terciarios.

\section{Procesos manifestados a través de la movilidad residencial}

El análisis anterior evidencia que los efectos de la movilidad residencial en la recomposición social de la ciudad difieren tanto en el tiempo como en el espacio. Respecto a la distribución espacial, se ha puesto de manifiesto que la movilidad residencial se relaciona de manera muy diferencial con el territorio urbano. Interpretando el territorio en función de las prácticas socioespaciales, 
se observa una implicación desigual de los barrios madrileños en los cambios residenciales y un cierto mantenimiento de estas diferencias en los dos periodos temporales analizados. Sin embargo, las repercusiones de esta movilidad en la recomposición social no son simples ni estables. Mientras que antes de 2008 un aumento de la movilidad residencial implicaba incrementos de la proporción de nacidos en el extranjero, un proceso de rejuvenecimiento más acuciado y un crecimiento del nivel educativo, a partir de 2008, esta influencia disminuye sustancialmente y la relación se vuelve más compleja, puesto que coexisten distintas relaciones entre la intensidad de la movilidad residencial y los efectos producidos. Estas diferencias entre uno y otro periodo se deben a una combinación de las modificaciones en el espacio social del municipio de Madrid, de las prácticas residenciales relacionadas con el cambio de vivienda de algunos grupos sociales, y a las prácticas socioespaciales de los mismos.

En primer lugar, las modificaciones en el espacio social madrileño producen modificaciones en el tamaño de diferentes grupos sociales definidos en cuanto a la edad, al país de nacimiento o al nivel educativo. Esa situación conlleva, por efectos del tamaño de la población, que haya una mayor participación de adultos, población nacida en el extranjero, o personas con estudios superiores en los cambios de vivienda, simplemente por el hecho de ser más numerosos, y no necesariamente por un cambio en los hábitus o en las prácticas residenciales asociados a estos grupos. De hecho, las modificaciones en las prácticas residenciales en relación con el traslado de vivienda caminan en sentido inverso. Respecto a la edad, esta se ha intensificado, especialmente entre los jóvenes o durante los primeros años del grupo adulto. En lo que atañe a la población nacida en el extranjero, se aprecia una reducción en el número de cambios residenciales, si bien estos siguen implicando un número muy superior a los que protagoniza la población nacida en España. La combinación del aumento de personas nacidas en el extranjero y sus prácticas residenciales, caracterizadas por un grado elevadísimo de desplazamientos, conlleva una alta participación de los mismos en la movilidad madrileña. Los resultados se relacionan con estudios que muestran una movilidad elevada entre colectivos vulnerables (Kingsley et al., 2012; Wiesel, 2014) concretamente de la población extranjera (Bayona y López-Gay, 2011; Bayona y Pujadas, 2010).

En último lugar, se aprecia una mutación social respecto al nivel educativo, protagonizada por una disminución de personas sin estudios y un aumento de aquellas con estudios superiores, lo que provoca una mayor participación de estas últimas en los movimientos residenciales que afectan al municipio. Sin embargo, respecto a las prácticas residenciales de los grupos por nivel educativo, solo se han intensificado los desplazamientos entre aquellos con menor nivel educativo, lo que puede indicar un aumento de la movilidad residencial más relacionada a situaciones de precariedad o de desposesión. Los efectos de esas modificaciones en las prácticas de algunos colectivos (por su mayor o menor representación en el espacio social o por una modificación de sus prácticas residenciales) conllevan manifestaciones diferenciales en la recomposición social del territorio madrileño. 
La falta de efectos lineales entre la movilidad residencial y los indicadores de variación de la composición social analizados en el segundo periodo podría manifestar una modificación de las prácticas espaciales por un aumento de aquellas más restringidas en el espacio, es decir, a un aumento de movilidad de proximidad espacial con un mayor protagonismo de los movimientos que se producen dentro del mismo barrio. Estos cambios residenciales computarían en los indicadores de movilidad, pero no producirían transformaciones en la composición social del barrio en el que se producen. Sin embargo, la reducción de las transformaciones asociadas a la movilidad residencial en el segundo periodo analizado parece deberse más a un aumento de la movilidad de proximidad socioespacial, es decir, a un incremento de los movimientos entre barrios de composición social similar ${ }^{21}$, o bien a que las entradas y las salidas de los barrios son llevadas a cabo por agentes sociales de características parecidas.

En términos generales, la movilidad contribuye en mayor medida a la transformación de la composición social en el primer periodo que en el segundo, lo que nos lleva a pensar en una creciente especialización socioespacial de las prácticas residenciales. En cualquier caso, la dispersión de sus efectos evidencia patrones de movilidad diferenciados que pueden encontrarse relacionados con diferentes procesos urbanos:

1. Se ha manifestado un grupo de barrios con alta movilidad y altos porcentajes de extranjeros y una reducción de la población con estudios superiores, lo que podría indicar la consolidación de barrios especializados en colectivos peor posicionados en el campo residencial con altos niveles de rotación.

2. Por otro lado, destacan los barrios receptores, que han experimentado una disminución de personas mayores, importantes aumentos de menores y de adultos, un incremento significativo del nivel educativo y una muy reducida proporción de personas nacidas en el extranjero, lo que parece relacionarse con la instalación de perfiles tradicionalmente relacionados con los procesos de suburbanización metropolitana.

3. Además, se ha evidenciado la diferente relación entre la intensidad de la movilidad y los procesos de transformación socioespacial en el segundo periodo, por lo que se pueden apreciar dos subgrupos dentro de los barrios de alta movilidad. En algunos de ellos, específicamente en los del Centro, se producen débiles incrementos o incluso reducciones de la población nacida en el extranjero y un aumento de población con estudios superiores, lo que podría ser producto de una reapropiación del espacio relacionada con procesos de gentrificación en el centro histórico madrileño, lo que implica la desposesión por parte de los peor posicionados.

21. A fin de corroborar esta idea, se ha realizado el mismo análisis mediante una Tasa de Reemplazo, que elimina los movimientos producidos dentro de un mismo barrio, con lo que se obtienen resultados muy similares a los recogidos con la Tasa de Movilidad. Tasa de reemplazo $=\frac{\left(\text { Entradas }^{t, t+n}+\text { Salidas }^{t, t+n}\right) / n}{\frac{1}{2} *\left(P o b^{t}+P o b^{t, t+n}\right)} * 1000$ 
4. Respecto a los barrios de media y baja movilidad, también aparecen diferentes patrones de transformación, especialmente en el segundo periodo. Se encuentra un grupo de barrios de baja movilidad ligados a procesos de envejecimiento, mientras que otros no están experimentando procesos de envejecimiento tan acuciados. Sería necesario comprobar si estas diferencias se deben a la disponibilidad o a la indisponiblidad de vivienda vacante, o a la capacidad de estos barrios para suponer un mercado para determinados grupos sociales.

\section{Conclusiones}

En el presente artículo, se ha desarrollado una propuesta de uso de la movilidad residencial para evaluar su relación y sus efectos en la composición social del espacio urbano.

A partir del caso madrileño, se ha realizado un análisis a escala inframunicipal (escasamente trabajada hasta ahora en España) sobre la movilidad residencial y sus efectos en la (re)producción social de los barrios que conforman el municipio. La fuente utilizada presenta ventajas e inconvenientes que han de ser tenidos en cuenta a la hora de interpretar los resultados. La principal ventaja es la posibilidad de llevar a cabo un análisis desagregado espacial y temporalmente, así como la consideración a nivel inframunicipal de todos los desplazamientos residenciales, tanto de entradas como de salidas, que hayan sido registrados. Como inconveniente principal, destaca la limitada información que aporta para el estudio de la composición social del espacio urbano. En cualquier caso, y a pesar de esta limitación, sí ha sido posible identificar algunas cuestiones interesantes y de relevancia para la comprensión de la dinámica urbana.

En cuanto a la dimensión territorial, se ha manifestado una gran estabilidad en la clasificación de los barrios en función de la intensidad de las prácticas relacionadas con el cambio de vivienda que les afectan. Sin embargo, los efectos de esta movilidad en la transformación de la composición social varían entre los periodos anterior y posterior al inicio de la crisis. Mientras que, en el primer periodo analizado, una mayor movilidad se relaciona con cambios en su composición social (aumento de la población nacida en el extranjero, rejuvenecimiento e incremento del nivel educativo), en el segundo, la movilidad residencial parece relacionarse más con una reproducción de la división social del espacio urbano que con una modificación del mismo.

Estas diferencias temporales se deben en parte a una modificación en los agentes sociales con mayor participación en los cambios de residencia. Algunas son provocadas por un cambio en el espacio social (mayor peso relativo entre los residentes en el municipio de las personas nacidas en el extranjero, de los adultos respecto a los jóvenes y de aquellas con estudios superiores) y otras, relacionadas con una modificación en las prácticas residenciales.

Sin embargo, la diferencia entre ambos periodos parece deberse, fundamentalmente, a un incremento de la proximidad social de las prácticas territoriales 
relacionada con una especialización de los barrios madrileños. Es decir, los desplazamientos residenciales de los agentes sociales ya no conllevan transformaciones en los barrios que implican, lo que indica que se estarían produciendo entre espacios de una composición social similar. En cualquier caso, a partir de 2008, se aprecia una mayor complejidad y la coexistencia de diferentes procesos de transformación de la composición social del espacio urbano, lo cual evidencia que la movilidad residencial, en sí misma, no cuenta con carácter transformador, pero que parece encontrarse asociada a procesos urbanos de carácter más general, suponiendo un elemento esencial para la interpretación de la dinámica urbana.

\section{Referencias bibliográficas}

ANDÚJAR, Andrea y FERIA, José María (2015). Residential vulnerability in Andalusian metropolitan areas: $A$ housing quality analyse. Lisboa: ENHR Conference.

AuTHIER, Jean-Yves (2014). «Préambule: Les trajectoires résidentielles, un champ de recherche pour saisir le sens des mobilités». En: Fol, Silvie; Miot, Yoan y ViGNAL, Cécile (dir.). Mobilités résidentielles, territoires et politiques publiques [en línea]. Villeneuve d'Ascq: Presses Universitaires du Septentrion. <https://doi.org/10.4000/books.septentrion.3182>.

BAYONA, Jordi; LOPEZ-GAY, Antonio (2011). "Concentración, segregación y movilidad residencial de los extranjeros en Barcelona». Documents d'Anàlisi Geogràfica [en línea], 57 (3), 381-412. <https://doi.org/10.5565/rev/dag.234>.

BAYONA, Jordi y PUJADAS, Isabel (2010). "Cambios residenciales internos en la ciudad de Barcelona: Evolución y características territoriales». Investigaciones Geográficas [en línea], 52, 9-36. $<$ https://doi.org/10.14198/ingeo2010.52.01>.

Beer, Andrew; Faulkner, Debbie y Gabriel, Michelle (2006). "21st Century Housing careers and Australia's housing future: Literature review». AHURI Research Paper, NRV2-1. Melbourne: Australian Housing and Urban Research Institute Limited.

Bourdieu, Pierre (1988). Cosas dichas. Buenos Aires: Gedisa.

- (1997). Razones prácticas: Sobre la teoría de la acción. Barcelona: Anagrama.

- (1999). «Los efectos del lugar». En: Bourdieu, Pierre (dir.). La miseria del mundo. Madrid: Akal, 119-124.

Bourdieu, Pierre; Bouhedja, Salah; Christin, Rosine y Givry, Claire (1990). «Un placement de père de famille [La maison individuelle: Spécificité du produit et logique du champ de production]». Actes de la Recherche en Sciences Sociales [en línea], 81-82, 6-33. <https://doi.org/10.3406/arss.1990.2924>.

Bourdieu, Pierre y Christin, Rosine (1990). «La construction du marché [Le champ administratif et la production de la "politique du logement"]». Actes de la Recherche en Sciences Sociales [en línea], 81 (1), 65-85. <https://doi.org/10.3406/arss.1990.2927>.

Bourdieu, Pierre y SAINT MARTIN, Monique de (1990). «Le sens de la propriété [La genèse sociale des systèmes de préférences]». Actes de la Recherche en Sciences Sociales [en línea], 81-82, 52-64.

<https://doi.org/10.3406/arss.1990.2926>. 
BOURDIEU, Pierre y WACQUANT, Loïc (2005). Una invitación a la sociología reflexiva. Buenos Aires: Siglo XXI Editores.

Brown, Lawrence A. y Moore, Eric G. (1970). «The Intra-Urban Migration Process: A Perspective». Geografiska Annaler: Series B, Human Geography [en línea], 52 (1), 1-13. $<$ https://doi.org/10.2307/490436>.

ClARK, William A.V. (2012). «Residential mobility and the housing market». En: Clapham, David; Clark, William A.V.; GibB, Kenneth (eds.). The Sage handbook of housing studies [en línea]. SAGE Publications Ltd. <http://dx.doi.org/10.4135/9781446247570.n4>.

Clark, William A.V. y Dieleman, Frans (1996). Households and Housing: Choice and Outcomes in the Housing Market. New Brunswick: Center for Urban Policy Research.

Clark, William A.V.; HAM, Maarten van y COUlTER, Rory (2014). «Spatial mobility and social outcomes». Journal of Housing and the Built Environment [en línea], 29 (4), 699-727. <https://doi.org/10.1007/s10901-013-9375-0>.

Clark, William A.V. y OnAKA, Jun L. (1983). «Life cycle and housing adjustment as explanations of residential mobility». Urban Studies [en línea], 20, 47-57. <https://doi.org/10.1080/713703176>.

Colectivo IOÉ (coord.) (2005). Inmigración y vivienda en España. Madrid: Ministerio de Trabajo y Asuntos Sociales.

CORTÉS, Luis (1995). La cuestión residencial: Bases para una sociología del habitar. Madrid: Fundamentos.

Cortés, Luis; MenéndeZ, Maria Victoria y NAVArRete, Jimena (2004). «La vivienda como factor de integración social en los inmigrantes». Documentación Social, 132, 121-156.

Courgeau, Daniel (1988). «Méthodes de mesure de la mobilité spatiale: Migrations internes, mobilité temporaire et navettes». Institut National d'Études Démographiques [en línea], 43 (4), 877-880. <https://doi.org/10.2307/1533496>.

Di VirgiLio, Maria Mercedes (2011). «La movilidad residencial: Una preocupación sociológica». Territorios, 25, 173-190.

- (2014) «Diferencias sociales en los procesos de movilidad residencial intraurbana en el Área Metropolitana de Buenos Aires (Argentina)». Quivera, 16 (1), 11-37.

DonZELOT, Jaques (2007). «La ciudad de tres velocidades». En: VVAA. La fragilización de las relaciones sociales. Madrid: Círculo de Bellas Artes.

DUHAU, Emilio (2003). «División social del espacio metropolitano y movilidad residencial». Papeles de Población, 9 (36), 161-210.

Dupont, Véronique; Dureau, Françoise; Lelièvre, Éva; LÉvy, Jean-Pierre; Lulle, Thierry (2002). «Introducción general». En: DuREAU, Françoise; DuPONT, Véronique; Lelièvre, Éva; LÉvY, Jean-Pierre y Lulle, Thierry (dir.). Metrópolis en movimiento. Bogotá: Alfaomega Colombiana.

Everaers, Pieter C.J. y MaAs, M.W. (1985). «Neighborhood change: Intra-urban migration and changing neighborhood use». Espace, Populations, Sociétés [en línea], 3 (1), 189-200. <https://doi.org/10.3406/espos.1985.1028>.

FERIA, José María (2010). «La movilidad residencial y los procesos de urbanización metropolitanos en España». En: Feria, José María y Albertos, Juan Miguel (coords.). La ciudad metropolitana en España: Procesos urbanos en los inicios del siglo XXI. Pamplona: Thomson Reuters-Civitas. 
- (coord.) (2008). Migraciones y movilidad residencial en Andalucía. Sevilla: Instituto de Estadística de Andalucía.

Form, William H. (1954). "The Place of Social Structure in the Determination of Land Use: Some Implications for a Theory of Urban Ecology». Social Forces [en línea], 32 (4), 317-323. <https://doi.org/10.2307/2574112>.

FORREST, Ray (1987). «Spatial Mobility, Tenure Mobility and Emerging Social Divisions in the UK Housing Market». Environment and Planning A [en línea], 19, 1611-1630. <https://doi.org/10.1068/a191611>.

Gale, Dennis. E. (1979). «Middle Class Resettlement in Older Urban Neighborhoods: The Evidence and the Implications». Journal of the American Planning Association [en línea], 45 (3), 293-304. <https://doi.org/10.1080/01944367908976968>.

Ham, Maarten van y Clark, William A.V. (2009). «Neighbourhood mobility in context: Household moves and changing neighbourhoods in the Netherlands». Environment and Planning $A$ [en línea], 41 (6), 1442-1459. <https://doi.org/10.1068/a4182>.

HarveY, David (1977). Urbanismo y desigualdad social. Madrid: Siglo XXI de España Editores, 2016.

Hedman, Lina (2011). «Residential Mobility and Neighbourhood Effects: A Holistic Approach». Geografiska Regionstudier, 88.

Hernández, Manuel y López, Diego Pascual (2013). "Condición inmigrante y exclusión residencial». En: HERNÁNDEZ PEDREÑO, Manuel (coord.). Vivienda y exclusión residencial. Murcia: Editum. Ediciones de la Universidad de Murcia.

KARSTEN, Lia (2007). «Housing as a Way of Life: Towards an Understanding of Middle-Class Families' Preference for an Urban Residential Location». Housing Studies [en línea], 22 (1), 83-98. <http://dx.doi.org/10.1080/02673030601024630>.

Kempen, Ronald van (2002). "The Academic Formulations: Explanations for the Partitioned City». En: Marcuse, Peter y Kempen, Ronald van (eds.). Of States and Cities: The Partitioning of the Urban Space. Nueva York: Oxford University Press.

KENDiG, Hal L. (1990). "A life course perspective on housing attainment». En: MYERS, Dowell (ed.). Housing demography: Linking demographic structure and housing markets. Madison: University of Wisconsin. Social Demography.

Kingsley, G. Thomas; Jordan, Audrey y TraynOr, William (2012). «Addressing Residential Instability: Options for Cities and Community Initiatives». Cityscape: A Journal of Policy Development and Research, 14 (3), 161-184.

LEAL, Jesús (2002). "Segregación social y mercados de vivienda en las grandes ciudades». RES: Revista Española de Sociología, 2, 59-75.

LÉVY, Jean-Pierre (1998). «Habitat et habitants: Position et mobilité dans l'espace résidentiel». En: Grafmeyer, Yves y Dansereau, Francine (dir.). Trajectories familiales et espaces de vie en milieu urbain. Lyon: Presses Universitaires de Lyon.

LeVY, Jean Pierre y Brun, Jacques (2002). «De la extensión a la renovación metropolitana: mosaico social y movilidad». En: Dureau, Françoise; DuPONT, Véronique; LELIÈVre, Éva; LÉVY, Jean-Pierre y Lulle, Thierry (coords.) Metrópolis en movimiento: Una comparación internacional, Bogotá, IRD Editions y Económica, Alfaomega Colombiana. 
LEY, David (1996). The new middle class and the remaking of the central city. Oxford: Oxford University Press.

LÓPEz, Cristina; PujADAS, Isabel y BAYONA, Jordi (2013). «Households within the residential mobility process: The case of the Barcelona metropolitan region». Archivio Di Studi Urbani E Regionali [en línea], 108, 57-81.

<https://doi.org/10.3280/asur2013-108004>.

LÓPEZ-GAY, Antonio (2016). «Atracción de talento y polarización socioeconómica en Barcelona». Perspectives Demogràfiques, 3, 1-4.

Martín Moreno, Jaime y RodrígueZ-Jaume, Maria José (2007). «El fenómeno de la migración en la sociedad de la precariedad: Análisis y medida». Sociedad y Utopía: Revista de Ciencias Sociales, 29, 521-548.

MÓDENES, Juan Antonio (1998). Flujos espaciales e itinerarios biográficos: La movilidad residencial en el área de Barcelona. Departamento de Geografía. Universitat Autònoma de Barcelona. Tesis doctoral.

Mulder, Clara H. (1993). Migration dynamics: A life course approach. Amsterdam: Netherlands Thesis Publishers.

Mulder, Clara H. y Hooimeijer, Pieter (1999). «Residential Relocations in the Life Course». En: Wissen, Leo van y Dykstra Pearl, A. (eds.). Population Issues: An Interdisciplinary Focus [en línea]. The Plenum Series on Demographic Methods and Population Analysis. <https://doi.org/10.1007/978-94-011-4389-9_6>.

Murie, A. (1997). «Placing housing in its social context». En: VESTERGAARD, H. (ed.). Housing in Europe. Horsholm: Statens Byggeforskningsinstitut.

PUJADAS, Isabel (2005). «De la ciudad compacta a la ciudad dispersa: Movilidad residencial en la Región Metropolitana de Barcelona 1982-2000». XXV International Population Conference, 20.

- (2009). «Movilidad residencial y expansión urbana en la región metropolitana de Barcelona». Scripta Nova: Revista Electrónica de Geografía y Ciencias Sociales, XIII (290).

Rodríguez MERKel, G.M. (2014). «Qué es y qué no es segregación residencial: Contribuciones para un debate pendiente». Biblio 3W: Revista Bibliográfica de Geografía y Ciencias Sociales [en línea], XIX (1079). <http://www.ub.es/geocrit/b3w-1079.htm>.

Rossi, Peter H. (1955). Why Families Move: A Study in the Social Psychology of Urban Residential Mobility. Glencoe: Free Press.

SABATINI, Francisco; CÁCERES, Gonzalo, y CerdÁ, Jorge. (2001). «Segregación residencial en las principales ciudades chilenas: Tendencias de las tres últimas décadas y posibles cursos de acción». EURE (Santiago), 27(82), 21-42. <https://dx.doi.org/10.4067/S0250-71612001008200002>

SHARKEY, Patrick (2012). "Residential Mobility and the Reproduction of Unequal Neighborhoods». Cityscape, 14 (3), 9-31.

Slater, Tom (2013). "Your Life Chances Affect Where You Live: A Critique of the "Cottage Industry" of Neighbourhood Effects Research». International Journal of Urban and Regional Research [en línea], 37 (2), 367-387. <https://doi.org/10.1111/j.1468-2427.2013.01215.x>.

SMITH, Neil (1979). «Toward a Theory of Gentrification a Back to the City Movement by Capital, not People». Journal of the American Planning Association [en línea], 45 (4), 538-548. <https://doi.org/10.1080/01944367908977002>. 
SORANDO, Daniel (2015). Espacios en conflicto: Un análisis relacional del cambio social en los centros estigmatizados. Departamento de Sociología II. Universidad Complutense de Madrid. Tesis doctoral.

Susino, Joaquín (2003). Movilidad residencial: Procesos demográficos, estrategias familiares y estructura social. Tesis doctoral.

- (2010). «La movilidad residencial diferencial en la reconfiguración metropolitana». En: FerIA, José María y AlberTos, Juan Miguel (coords.). La ciudad metropolitana en España: Procesos urbanos en los inicios del siglo XXI. Pamplona: Thomson ReutersCivitas.

Theodore, Nik; Peck, Jamie y Brenner, Neil (2009). «Urbanismo neoliberal: La ciudad y el imperio de los mercados». Temas Sociales SUR, 12.

Vignal, Cécile (2014). "L'ancrage local, une ressource pour les classes populaires des territoires désindustrialisés». En: Fol, Silvie; Miot, Yoan y Vignal, Cécile (dir.). Mobilités résidentielles, territoires et politiques publiques [en línea]. Villeneuve d'Ascq: Presses Universitaires du Septentrion. <https://doi.org/10.4000/books.septentrion.3203>.

Vinuesa, Julio (coord.); Zamora, Francisco; GÉnOva, Ricard; Serrano, Pedro y RECAÑo, Joaquín (1994). Demografía: Análisis y proyecciones. Madrid: Síntesis.

Wiesel, I. (2014). "Mobilities of Disadvantage: The Housing Pathways of Low-income Australians». Urban Studies [en línea], 51 (2), 319-334. <https://doi.org/10.1177/0042098013489739>.

WiLKIS, A. (2004). «Apuntes sobre la noción de estrategia en Pierre Bourdieu». Revista Argentina de Sociología, 2 (3), 118-130.

\section{Anexo}

Tabla 1. Barrios resultantes de los análisis de conglomerados por el método de K-medias en los dos periodos analizados

\begin{tabular}{|c|c|c|c|c|c|}
\hline \multirow{2}{*}{\multicolumn{2}{|c|}{ Tipología de barrios según movilidad }} & \multicolumn{2}{|c|}{$2000-2007$} & \multicolumn{2}{|c|}{ 2008-2013 } \\
\hline & & $\mathrm{N}$ & Media & $\mathrm{N}$ & Media \\
\hline \multirow[t]{3}{*}{ Baja movilidad } & Tasa de Entradas Totales & 56 & 72,46 & 52 & 72,13 \\
\hline & Tasa de Salidas Totales. & 56 & 68,66 & 52 & 69,89 \\
\hline & Tasa de Movimientos Intrabarrio. & 56 & 11,38 & 52 & 14,76 \\
\hline \multirow[t]{3}{*}{ Movilidad media } & Tasa de Entradas Totales. & 51 & 106,80 & 55 & 101,44 \\
\hline & Tasa de Salidas Totales. & 51 & 86,21 & 55 & 94,23 \\
\hline & Tasa de Movimientos Intrabarrio. & 51 & 15,77 & 55 & 20,68 \\
\hline \multirow[t]{3}{*}{ Movilidad alta } & Tasa de Entradas Totales. & 13 & 150,40 & 14 & 142,58 \\
\hline & Tasa de Salidas Totales. & 13 & 121,78 & 14 & 129,76 \\
\hline & Tasa de Movimientos Intrabarrio. & 13 & 20,70 & 14 & 31,98 \\
\hline \multirow[t]{3}{*}{ Barrios receptores } & Tasa de Entradas Totales. & 8 & 185,93 & 7 & 139,69 \\
\hline & Tasa de Salidas Totales. & 8 & 70,21 & 7 & 83,34 \\
\hline & Tasa de Movimientos Intrabarrio. & 8 & 9,79 & 7 & 18,07 \\
\hline \multirow[t]{3}{*}{ Total periodo } & Tasa de Entradas Totales. & 128 & 101,15 & 128 & 96,12 \\
\hline & Tasa de Salidas Totales. & 128 & 81,14 & 128 & 87,63 \\
\hline & Tasa de Movimientos Intrabarrio. & 128 & 13,98 & 128 & 19,37 \\
\hline
\end{tabular}

Fuente: elaboración propia a partir del Padrón Municipal de Habitantes. 


\section{Mapa 1. Barrios y códigos del municipio de Madrid}

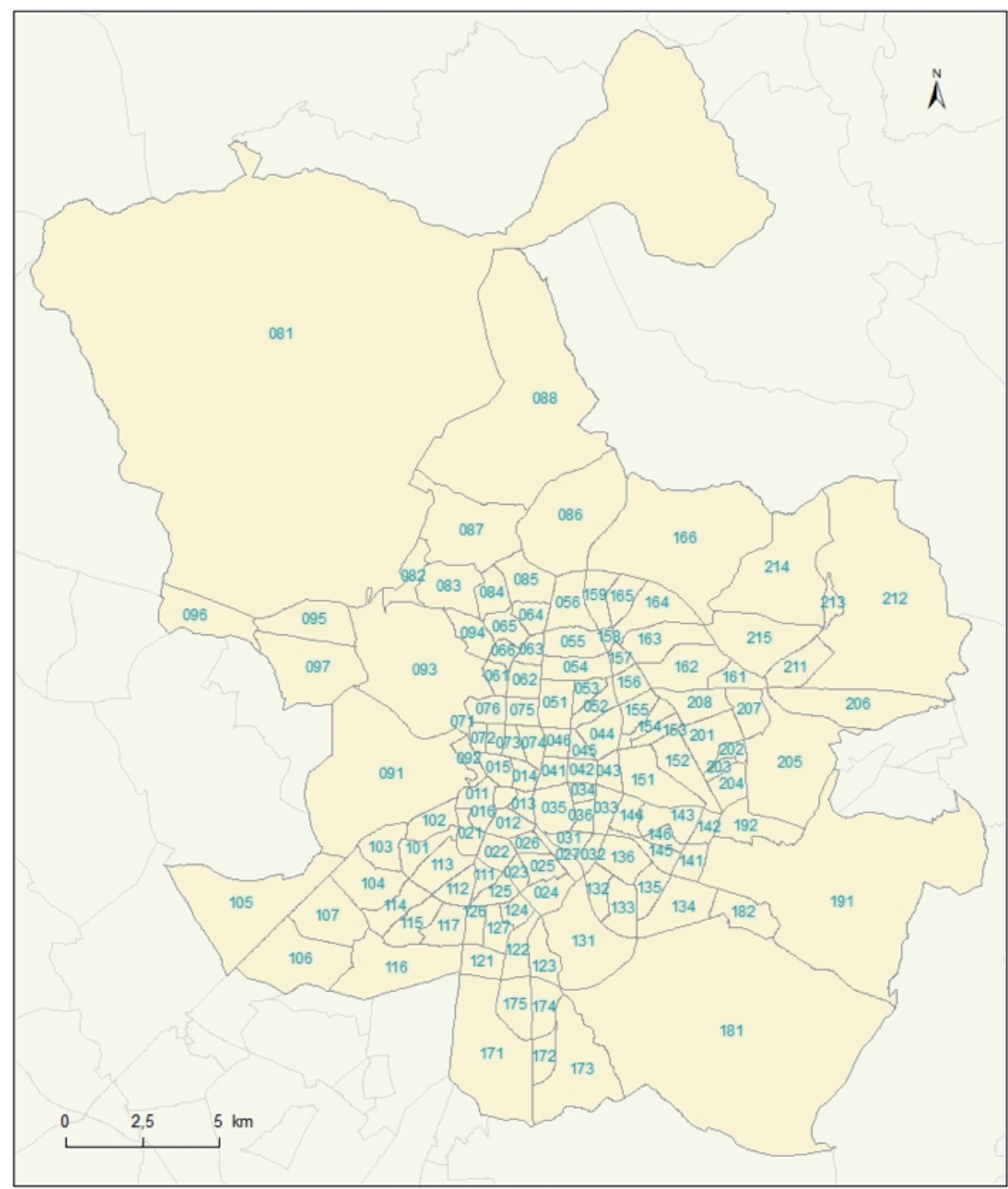

Fuente: elaboración propia a partir de la información del Ayuntamiento de Madrid. 
Tabla 1. Códigos y nombres de los distritos y de los barrios del municipio de Madrid

\author{
01. Centro

011. Palacio
012. Embajadores
013. Cortes
014. Justicia
015. Universidad
016. Sol

02. Arganzuela

021. Imperial

022. Acacias

023. Chopera

024. Legazpi

025. Delicias

026. Palos de Moguer

027. Atocha

\subsection{Retiro}

031. Pacífico

032. Adelfas

033. Estrella

034. Ibiza

035. Jerónimos

036. Niño Jesús

04. Salamanca

041. Recoletos

042. Goya

043. Fuente de Berro

044. Guindalera

045. Lista

046. Castellana

05. Chamartín

051. El Viso

052. Prosperidad

053. Ciudad Jardín

054. Hispanoamérica

055. Nueva España

056. Castilla

06. Tetuán

061. Bellas Vistas

062. Cuatro Caminos

063. Castillejos

064. Almenara

065. Valdeacederas

066. Berruguete

07. Chamberí

071. Gaztambide

072. Arapiles

073. Trafalgar

074. Almagro

075. Ríos Rosas

076. Vallehermoso
08. Fuencarral - El Pardo

081. El Pardo

082. Fuentelarreina

083. Peñagrande

084. Pilar

085. La Paz

086. Valverde

087. Mirasierra

088. El Goloso

09. Moncloa - Aravaca

091. Casa de Campo

092. Argüelles

093. Ciudad Universitaria

094. Valdezarza

095. Valdemarín

096. El Plantío

097. Aravaca

10. Latina

101. Los Cármenes

102. Puerta del Ángel

103. Lucero

104. Aluche

105. Campamento

106. Cuatro Vientos

107. Las Águilas

11. Carabanchel

111. Comillas

112. Opañel

113. San Isidro

114. Vista Alegre

115. Puerta Bonita

116. Buenavista

117. Abrantes

12. Usera

121. Orcasitas

122. Orcasur

123. San Fermín

124. Almendrales

125. Moscardó

126. Zofio

127. Pradolongo

13. Puente de Vallecas

131. Entrevías

132. San Diego

133. Palomeras Bajas

134. Palomeras Sureste

135. Portazgo

136. Numancia

14. Moratalaz

141. Pavones

142. Horcajo
143. Marroquina

144. Media Lengua

145. Fontarrón

146. Vinateros

15. Ciudad Lineal

151. Ventas

152. Pueblo Nuevo

153. Quintana

154. Concepción

155. San Pascual

156. San Juan Bautista

157. Colina

158. Atalaya

159. Costillares

16. Hortaleza

161. Palomas

162. Piovera

163. Canillas

164. Pinar del Rey

165. Apóstol Santiago

166. Valdefuentes

17. Villaverde

171. San Andrés

172. San Cristóbal

173. Butarque

174. Los Rosales

175. Los Ángeles

18. Villa de Vallecas

181. Casco Histórico de Vallecas

182. Santa Eugenia

19. Vicálvaro

191. Casco Histórico de Vicálvaro

192. Ambroz

20. San Blas - Canillejas 201. Simancas

202. Hellín

203. Amposta

204. Arcos

205. Rosas

206. Rejas

207. Canillejas

208. Salvador

21. Barajas

211. Alameda de Osuna

212 Aeropuerto

213. Casco Histórico de Barajas

214. Timón

215. Corralejos 